\title{
Bayesian post-processor and other enhancements of Subset Simulation for estimating failure probabilities in high dimensions
}

\author{
Konstantin M. Zuev ${ }^{\text {a,* }}$, James L. Beck ${ }^{a}$, Siu-Kui Au ${ }^{\text {b }}$, Lambros S. Katafygiotis ${ }^{c}$ \\ ${ }^{a}$ Division of Engineering and Applied Science, California Institute of Technology, Mail Code 104-44, Pasadena, CA 91125, USA \\ ${ }^{\mathrm{b}}$ Department of Building and Construction, City University of Hong Kong, 83 Tat Chee Avenue, Kowloon, Hong Kong \\ c Department of Civil and Environmental Engineering, The Hong Kong University of Science and Technology, Hong Kong, China
}

\section{A R T I C L E I N F O}

\section{Article history:}

Received 26 July 2011

Accepted 20 October 2011

Available online 21 November 2011

\section{Keywords:}

Reliability engineering

Stochastic simulation methods

Markov chain Monte Carlo

Subset Simulation

Bayesian approach

\begin{abstract}
A B S T R A C T
Estimation of small failure probabilities is one of the most important and challenging computational problems in reliability engineering. The failure probability is usually given by an integral over a highdimensional uncertain parameter space that is difficult to evaluate numerically. This paper focuses on enhancements to Subset Simulation (SS), proposed by Au and Beck, which provides an efficient algorithm based on MCMC (Markov chain Monte Carlo) simulation for computing small failure probabilities for general high-dimensional reliability problems. First, we analyze the Modified Metropolis algorithm (MMA), an MCMC technique, which is used in SS for sampling from high-dimensional conditional distributions. The efficiency and accuracy of SS directly depends on the ergodic properties of the Markov chains generated by MMA, which control how fast the chain explores the parameter space. We present some observations on the optimal scaling of MMA for efficient exploration, and develop an optimal scaling strategy for this algorithm when it is employed within SS. Next, we provide a theoretical basis for the optimal value of the conditional failure probability $p_{0}$, an important parameter one has to choose when using SS. We demonstrate that choosing any $p_{0} \in[0.1,0.3]$ will give similar efficiency as the optimal value of $p_{0}$. Finally, a Bayesian post-processor SS+ for the original SS method is developed where the uncertain failure probability that one is estimating is modeled as a stochastic variable whose possible values belong to the unit interval. Simulated samples from SS are viewed as informative data relevant to the system's reliability. Instead of a single real number as an estimate, SS+ produces the posterior PDF of the failure probability, which takes into account both prior information and the information in the sampled data. This PDF quantifies the uncertainty in the value of the failure probability and it may be further used in risk analyses to incorporate this uncertainty. To demonstrate SS+, we consider its application to two different reliability problems: a linear reliability problem and reliability analysis of an elasto-plastic structure subjected to strong seismic ground motion. The relationship between the original SS and SS+ is also discussed.
\end{abstract}

(c) 2011 Elsevier Ltd. All rights reserved.

\section{Introduction}

One of the most important and challenging problems in reliability engineering is to estimate the failure probability $p_{F}$ for a system, that is, the probability of unacceptable system performance. This is usually expressed as an integral over a high-dimensional uncertain parameter space:

$p_{F}=\int I_{F}(\theta) \pi(\theta) d \theta=\mathbb{E}_{\pi}\left[I_{F}(\theta)\right]$

\footnotetext{
* Corresponding author.

E-mail addresses: zuev@caltech.edu (K.M. Zuev), jimbeck@caltech.edu (J.L. Beck), siukuiau@cityu.edu.hk (S.-K. Au), lambros@ust.hk (L.S. Katafygiotis).
}

where $\theta \in \mathbb{R}^{d}$ represents the uncertain parameters needed to specify completely the excitation and dynamic model of the system; $\pi(\theta)$ is the joint probability density function (PDF) for $\theta ; F \subset \mathbb{R}^{d}$ is the failure domain in the parameter space (i.e. the set of parameter values that lead to performance of the system that is considered to be unacceptable); and $I_{F}(\theta)$ stands for the indicator function, i.e. $I_{F}(\theta)=1$ if $\theta \in F$ and $I_{F}(\theta)=0$ if $\theta \notin F$. The dimension $d$ is typically large for dynamic reliability problems (e.g. $d \sim 10^{3}$ ) because the stochastic input time history is discretized in time. As a result, the usual numerical quadrature methods for integrals are not computationally feasible for evaluation (1).

Over the past decade, the engineering research community has realized the importance of advanced stochastic simulation methods for reliability analysis. As a result, many different efficient algorithms have been developed recently, e.g. Subset Simulation [2], Importance Sampling using Elementary Events [3], Line 
Sampling [28], auxiliary domain method [25], Spherical Subset Simulation [24], Horseracing Simulation [44], to name but a few.

This paper focuses on enhancements to Subset Simulation (SS), proposed by Au and Beck [2], which provides an efficient algorithm for computing failure probabilities for general high-dimensional reliability problems. It has been shown theoretically [2] and verified with different numerical examples (e.g. $[4,5,38,39]$ ) that SS gives much higher computational efficiency than standard Monte Carlo Simulation when estimating small failure probabilities. Recently, various modifications of SS have been proposed: SS with splitting [9], Hybrid SS [10], and Two-Stage SS [23]. It is important to highlight, however, that none of these modifications offer a drastic improvement over the original algorithm.

We start with the analysis of the Modified Metropolis algorithm (MMA), a Markov chain Monte Carlo technique used in SS, which is presented in Section 2. The efficiency and accuracy of SS directly depends on the ergodic properties of the Markov chains generated by MMA. In Section 3, we examine the optimal scaling of MMA to tune the parameters of the algorithm to make the resulting Markov chain converge to stationarity as fast as possible. We present a collection of observations on the optimal scaling of MMA for different numerical examples, and develop an optimal scaling strategy for MMA when it is employed within SS for estimating small failure probabilities.

One of the most important components of SS which affects its efficiency is the choice of the sequence of intermediate threshold values or, equivalently, the intermediate failure probabilities (see Section 2, where the original SS algorithm is described). In Section 4 , a method for optimally choosing these probabilities is presented.

The usual interpretation of Monte Carlo methods is consistent with a purely frequentist approach, meaning that they can be interpreted in terms of the frequentist definition of probability which identifies it with the long-run relative frequency of occurrence of an event. An alternative interpretation can be made based on the Bayesian approach which views probability as a measure of the plausibility of a proposition conditional on incomplete information that does not allow us to establish the truth or falsehood of the proposition with certainty. Bayesian probability theory was, in fact, primarily developed by the mathematician and astronomer Laplace $[29,30]$ for statistical analysis of astronomical observations. Moreover, Laplace developed the well-known Bayes' theorem in full generality, while Bayes did it only for a special case [6]. A complete development based on Laplace's theory, with numerous examples of its applications, was given by the mathematician and geophysicist Jeffreys [22] in the early 20th century. Despite its usefulness in applications, the work of Laplace and Jeffreys on probability was rejected in favor of the frequentist approach by most statisticians until late last century. Because of the absence of a strong rationale behind the theory at that time, it was perceived as subjective and not rigorous by many statisticians. A rigorous logic foundation for the Bayesian approach was given in the seminal work of the physicist Cox [11,12] and expounded by the physicist Jaynes [20,21], enhancing Bayesian probability theory as a convenient mathematical language for inference and uncertainty quantification. Although the Bayesian approach usually leads to high-dimensional integrals that often cannot be evaluated analytically nor numerically by straightforward quadrature, the development of Markov chain Monte Carlo algorithms and increasing computing power have led over the past few decades to an explosive growth of Bayesian papers in all research disciplines.

In Section 5 of this paper, a Bayesian post-processor for the original Subset Simulation method is developed, where the uncertain failure probability that one is estimating is modeled as a stochastic variable whose possible values belong to the unit interval.
Although this failure probability is a constant defined by the integral in (1), its exact value is unknown because the integral cannot be evaluated; instead, we must infer its value from available relevant information. Instead of a single real number as an estimate, the post-processor, written as SS+ ("SS-plus") for short, produces the posterior PDF of the failure probability, which takes into account both relevant prior information and the information from the samples generated by SS. This PDF expresses the relative plausibility of each possible value of the failure probability based on this information. Since this PDF quantifies the uncertainty in the value of $p_{F}$, it can be fully used in risk analyses (e.g. for life-cycle cost analysis, decision making under risk, etc.), or it can be used to give a point estimate such as the most probable value based on the available information.

\section{Subset Simulation}

\subsection{Basic idea of Subset Simulation}

The original and best known stochastic simulation algorithm for estimating high-dimensional integrals is Monte Carlo Simulation (MCS). In this method the failure probability $p_{F}$ is estimated by approximating the mean of $I_{F}(\theta)$ in (1) by its sample mean:

$p_{F} \approx \hat{p}_{F}^{M C}=\frac{1}{N} \sum_{i=1}^{N} I_{F}\left(\theta^{(i)}\right)$

where samples $\theta^{(1)}, \ldots, \theta^{(N)}$ are independent and identically distributed (i.i.d.) samples from $\pi(\cdot)$, denoted $\theta^{(i)} \stackrel{i \text { i..d. }}{\sim} \pi(\cdot)$. This estimate is just the fraction of samples that produce system failure according to a model of the system dynamics. Notice that each evaluation of $I_{F}$ requires a deterministic system analysis to be performed to check whether the sample implies failure. The main advantage of MCS is that its efficiency does not depend on the dimension $d$ of the parameter space. Indeed, straightforward calculation shows that the coefficient of variation (c.o.v.) of the Monte Carlo estimate (2), serving as a measure of accuracy in the usual interpretation of MCS, is given by:

$\delta\left(\hat{p}_{F}^{M C}\right)=\sqrt{\frac{1-p_{F}}{N p_{F}}}$.

However, MCS has a serious drawback: it is inefficient in estimating small failure probabilities. If $p_{F}$ is very small, $p_{F} \ll 1$, then it follows from (3) that the number of samples $N$ (or, equivalently, number of system analyses) needed to achieve an acceptable level of accuracy is very large, $N \propto 1 / p_{F} \gg 1$. This deficiency of MCS has motivated research to develop more efficient stochastic simulation algorithms for estimating small failure probabilities in high-dimensions.

The basic idea of Subset Simulation [2] is the following: represent a very small failure probability $p_{F}$ as a product of larger probabilities so $p_{F}=\prod_{j=1}^{m} p_{j}$, where the factors $p_{j}$ are estimated sequentially, $p_{j} \approx \hat{p}_{j}$, to obtain an estimate $\hat{p}_{F}^{S S}$ for $p_{F}$ as $\hat{p}_{F}^{S S}=$ $\prod_{j=1}^{m} \hat{p}_{j}$. To reach this goal, let us consider a decreasing sequence of nested subsets of the parameter space, starting from the entire space and shrinking to the failure domain $F$ :

$\mathbb{R}^{d}=F_{0} \supset F_{1} \supset \cdots \supset F_{m-1} \supset F_{m}=F$.

Subsets $F_{1}, \ldots, F_{m-1}$ are called intermediate failure domains. As a result, the failure probability $p_{F}=P(F)$ can be rewritten as a product of conditional probabilities:

$p_{F}=\prod_{j=1}^{m} P\left(F_{j} \mid F_{j-1}\right)=\prod_{j=1}^{m} p_{j}$, 
where $p_{j}=P\left(F_{j} \mid F_{j-1}\right)$ is the conditional probability at the $(j-1)$ th conditional level. Clearly, by choosing the intermediate failure domains appropriately, all conditional probabilities $p_{j}$ can be made large. Furthermore, they can be estimated, in principle, by the fraction of independent conditional samples that cause failure at the intermediate level:

$p_{j} \approx \hat{p}_{j}^{M C}=\frac{1}{N} \sum_{i=1}^{N} I_{F_{j}}\left(\theta_{j-1}^{(i)}\right), \quad \theta_{j-1}^{(i)} \stackrel{\text { i.i.d. }}{\sim} \pi\left(\cdot \mid F_{j-1}\right)$.

Hence, the original problem (estimation of the small failure probability $p_{F}$ ) is replaced by a sequence of $m$ intermediate problems (estimation of the larger failure probabilities $p_{j}, j=1, \ldots, m$ ).

The first probability $p_{1}=P\left(F_{1} \mid F_{0}\right)=P\left(F_{1}\right)$ is straightforward to estimate by MCS, since (6) requires sampling from $\pi(\cdot)$ that is assumed to be readily sampled. However, if $j \geqslant 2$, to estimate $p_{j}$ using (6) one needs to generate independent samples from conditional distribution $\pi\left(\cdot \mid F_{j-1}\right)$, which, in general, is not a trivial task. It is not efficient to use MCS for this purpose, especially at higher levels, but it can be done by a specifically tailored Markov chain Monte Carlo technique at the expense of generating dependent samples.

Markov chain Monte Carlo (MCMC) [31,34,35] is a class of algorithms for sampling from multi-dimensional target probability distributions that cannot be directly sampled, at least not efficiently. These methods are based on constructing a Markov chain that has the distribution of interest as its stationary distribution. By simulating samples from the Markov chain, they will eventually be draws from the target probability distribution but they will not be independent samples. In Subset Simulation, the Modified Metropolis algorithm (MMA) [2], an MCMC technique based on the original Metropolis algorithm $[33,18]$, is used for sampling from the conditional distributions $\pi\left(\cdot \mid F_{j-1}\right)$.

Remark 1. It was observed in [2] that the original Metropolis algorithm does not work in high-dimensional conditional probability spaces, because it produces a Markov chain with very highly correlated states. The geometrical reasons for this are discussed in [26].

\subsection{Modified Metropolis algorithm}

Suppose we want to generate a Markov chain with stationary distribution

$\pi(\theta \mid \mathbb{F})=\frac{\pi(\theta) I_{\mathbb{F}}(\theta)}{P(\mathbb{F})}=\frac{\prod_{k=1}^{d} \pi_{k}\left(\theta_{k}\right) I_{\mathbb{F}}(\theta)}{P(\mathbb{F})}$

where $\mathbb{F} \subset \mathbb{R}^{d}$ is a subset of the parameter space. Without significant loss of generality, we assume here that $\pi(\theta)=\prod_{k=1}^{d} \pi_{k}\left(\theta_{k}\right)$, i.e. components of $\theta$ are independent (but are not so when conditioned on $\mathbb{F}$ ). MMA differs from the original Metropolis algorithm in the way the candidate state $\xi=\left(\xi_{1}, \ldots, \xi_{d}\right)$ is generated. Instead of using a $d$-variate proposal PDF on $\mathbb{R}^{d}$ to directly obtain the candidate state, in MMA a sequence of univariate proposal PDFs is used. Namely, each coordinate $\xi_{k}$ of the candidate state is generated separately using a univariate proposal distribution dependent on the coordinate $\theta_{k}$ of the current state. Then a check is made whether the $d$-variate candidate generated in such a way belongs to the subset $\mathbb{F}$ in which case it is accepted as the next Markov chain state; otherwise it is rejected and the current MCMC sample is repeated. To summarize, the Modified Metropolis algorithm proceeds as follows:

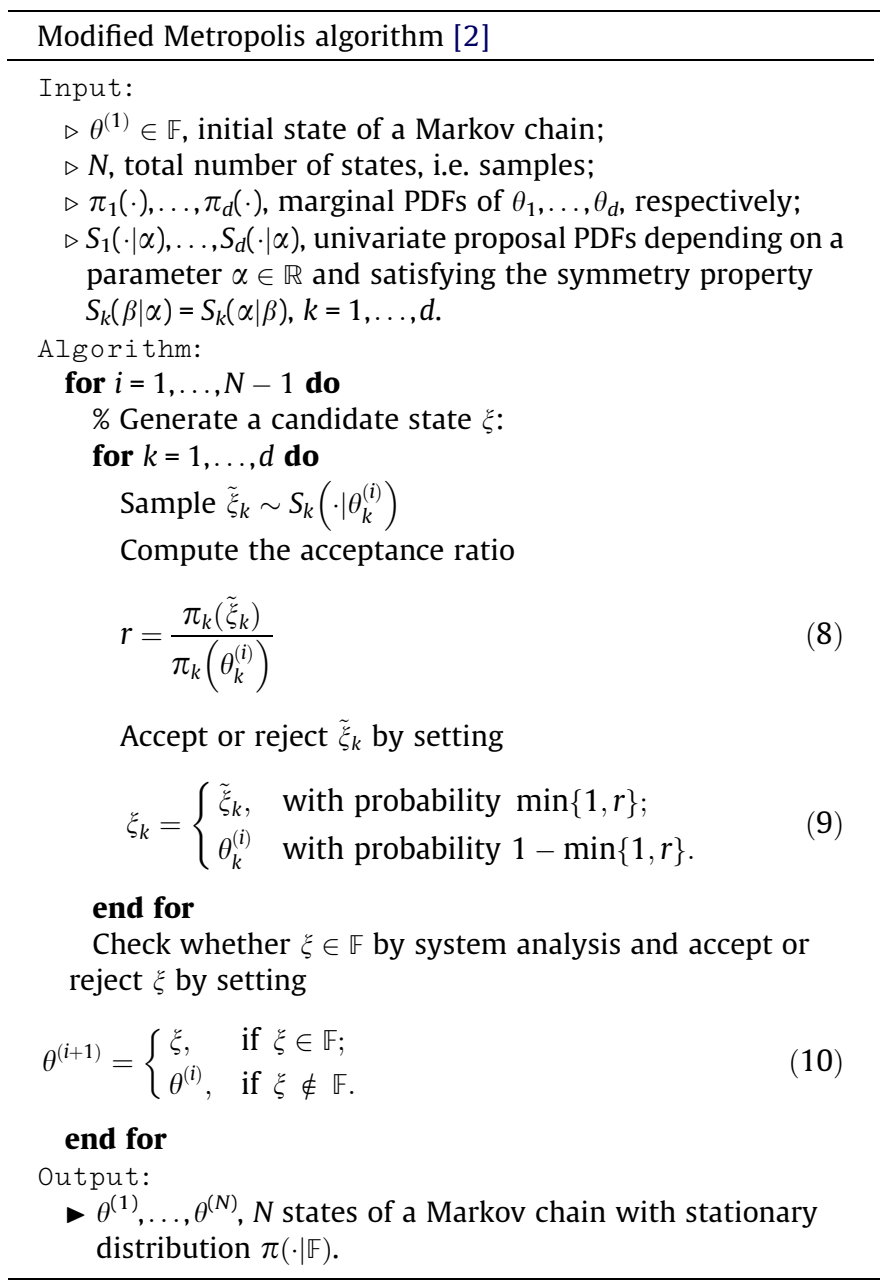

Schematically, the Modified Metropolis algorithm is shown in Fig. 1. For completeness and the reader's convenience, the proof that $\pi(\cdot \mid \mathbb{F})$ is the stationary distribution for the Markov chain generated by MMA is given in the appendix.

Remark 2. The symmetry property $S_{k}(\beta \mid \alpha)=S_{k}(\alpha \mid \beta)$ does not play a critical role. If $S_{k}$ does not satisfy this property, by replacing the "Metropolis" ratio in (8) by the "Metropolis-Hastings" ratio

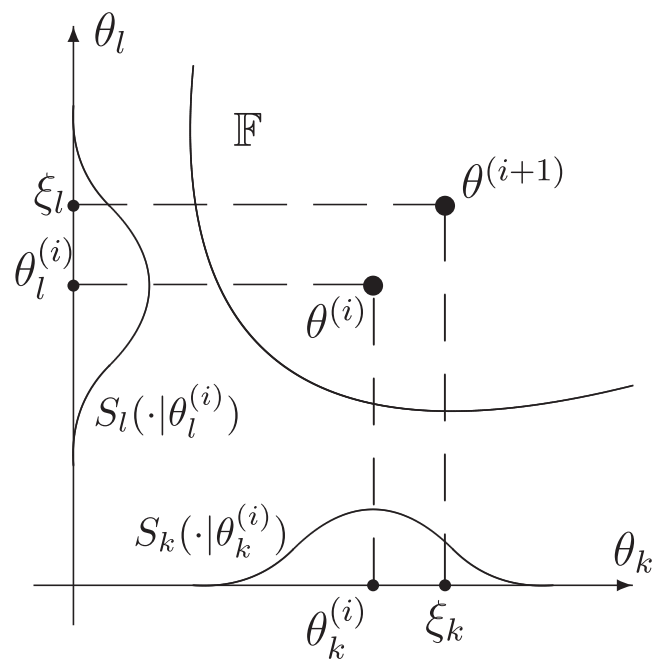

Fig. 1. Modified Metropolis algorithm. 
$r=\frac{\pi_{k}\left(\tilde{\xi}_{k}\right) S_{k}\left(\theta_{k}^{(i)} \mid \tilde{\xi}_{k}\right)}{\pi_{k}\left(\theta_{k}^{(i)}\right) S_{k}\left(\tilde{\xi}_{k} \mid \theta_{k}^{(i)}\right)}$,

we obtain an MCMC algorithm referred to as the Modified Metropolis-Hastings algorithm.

Thus, if we run the Markov chain for sufficiently long (the burnin period), starting from essentially any "seed" $\theta^{(1)} \in \mathbb{F}$, then for large $N$ the distribution of $\theta^{(N)}$ will be approximately $\pi(\cdot \mid \mathbb{F})$. Note, however, that in any practical application it is very difficult to check whether the Markov chain has reached its stationary distribution. If the seed $\theta^{(1)} \sim \pi(\cdot \mid \mathbb{F})$, then all states $\theta^{(i)}$ will be automatically distributed according to the target distribution, $\theta^{(i)} \sim \pi(\cdot \mid \mathbb{F})$, since it is the stationary distribution for the Markov chain. This is called perfect sampling [35] and Subset Simulation has this property because of the way the seeds are chosen [2].

Let us assume now that we are given a seed $\theta_{j-1}^{(1)} \sim \pi\left(\cdot \mid F_{j-1}\right)$, where $j=2, \ldots, m$. Then, using MMA, we can generate a Markov chain with $N$ states starting from this seed and construct an estimate for $p_{j}$ similar to (6), where MCS samples are replaced by MCMC samples:

$p_{j} \approx \hat{p}_{j}^{M C M C}=\frac{1}{N} \sum_{i=1}^{N} I_{F_{j}}\left(\theta_{j-1}^{(i)}\right), \quad \theta_{j-1}^{(i)} \sim \pi\left(\cdot \mid F_{j-1}\right)$.

Note that all samples $\theta_{j-1}^{(1)}, \ldots, \theta_{j-1}^{(N)}$ in (12) are identically distributed in the stationary state of the Markov chain, but are not independent. Nevertheless, these MCMC samples can be used for statistical averaging as if they were i.i.d., although with some reduction in efficiency [14]. Namely, the more correlated $\theta_{j-1}^{(1)}, \ldots, \theta_{j-1}^{(N)}$ are, the less efficient is the estimate (12). The correlation between successive samples is due to proposal PDFs $S_{k}$, which govern the generation of the next state of the Markov chain from the current one in MMA. Hence, the choice of proposal PDFs $S_{k}$ controls the efficiency of estimate (12), making this choice very important. It was observed in [2] that the efficiency of MMA depends on the spread of proposal distributions, rather then on their type. Both small and large spreads tend to increase the dependence between successive samples, slowing the convergence of the estimator. Large spreads may reduce the acceptance rate in (10), increasing the number of repeated MCMC samples. Small spreads, on the contrary, may lead to a reasonably high acceptance rate, but still produce very correlated samples due to their close proximity. To find the optimal spread of proposal distributions for MMA is a non-trivial task which is discussed in Section 3.

Remark 3. In [45] another modification of the Metropolis algorithm, called Modified Metropolis-Hastings algorithm with Delayed Rejection (MMHDR), has been proposed. The key idea behind MMHDR is to reduce the correlation between states of the Markov chain. A way to achieve this goal is the following: whenever a candidate $\xi$ is rejected in (10), instead of getting a repeated sample $\theta^{(i+1)}=\theta^{(i)}$, as the case of MMA, a new candidate $\tilde{\xi}$ is generated using a new set of proposal PDFs $\widetilde{S}_{k}$. Of course, the acceptance ratios (8) for the second candidate have to be adjusted in order to keep the target distribution stationary. In general, MMHDR generates less correlated samples than MMA but it is computationally more expensive.

\subsection{Subset Simulation algorithm}

Subset Simulation uses the estimates (6) for $p_{1}$ and (12) for $p_{j}$, $j \geqslant 2$, to obtain the estimate for the failure probability:

$p_{F} \approx \hat{p}_{F}^{S S}=\hat{p}_{1}^{M C} \prod_{j=2}^{m} \hat{p}_{j}^{M C M C}$
The remaining ingredient of Subset Simulation that we have to specify is the choice of intermediate failure domains $F_{1}, \ldots, F_{m-1}$. Usually, performance of a dynamical system is described by a certain positive-valued performance function $g: \mathbb{R}^{d} \rightarrow \mathbb{R}_{+}$, for instance, $g(\theta)$ may represent some peak (maximum) response quantity when the system model is subjected to the uncertain excitation $\theta$. Then the failure region, i.e. unacceptable performance region, can be defined as a set of excitations that lead to the exceedance of some prescribed critical threshold $b$ :

$F=\left\{\theta \in \mathbb{R}^{d}: g(\theta)>b\right\}$.

The sequence of intermediate failure domains can then be defined as

$F_{j}=\left\{\theta \in \mathbb{R}^{d}: g(\theta)>b_{j}\right\}$,

where $0<b_{1}<\cdots<b_{m-1}<b_{m}=b$. Intermediate threshold values $b_{j}$ define the values of the conditional probabilities $p_{j}=P\left(F_{j} \mid F_{j-1}\right)$ and, therefore, affect the efficiency of Subset Simulation. In practical cases it is difficult to make a rational choice of the $b_{j}$-values in advance, so the $b_{j}$ are chosen adaptively (see (16)) so that the estimated conditional probabilities are equal to a fixed value $p_{0} \in(0,1)$. We will refer to $p_{0}$ as the conditional failure probability.

Subset Simulation algorithm [2

Input:

$\triangleright p_{0}$, conditional failure probability;

$\triangleright N$, number of samples per conditional level.

Algorithm:

Set $j=0$, number of conditional level

Set $N_{F}(j)=0$, number of failure samples at level $j$

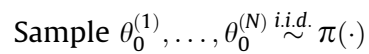

for $i=1, \ldots, N$ do

if $g^{(i)}=g\left(\theta_{0}^{(i)}\right)>b$ do

$N_{F}(j) \leftarrow N_{F}(j)+1$

end if

end for

while $N_{F}(j) / N<p_{0}$ do

$j \leftarrow j+1$

Sort $\left\{g^{(i)}\right\}: g^{\left(i_{1}\right)} \leqslant g^{\left(i_{2}\right)} \leqslant \cdots \leqslant g^{\left(i_{N}\right)}$

Define

$b_{j}=\frac{g^{\left(i_{N-N p_{0}}\right)}+g^{\left(i_{N-N p_{0}+1}\right)}}{2}$

for $k=1, \ldots, N p_{0}$ do

Starting from $\theta_{j}^{(1), k}=\theta_{j-1}^{\left(i_{N-N p_{0}+k}\right)} \sim \pi\left(\cdot \mid F_{j}\right)$, generate $1 / p_{0}$ states of a Markov chain $\theta_{j}^{(1), k}, \ldots, \theta_{j}^{\left(1 / p_{0}\right), k} \sim \pi\left(\cdot \mid F_{j}\right)$, using MMA.

end for

Renumber: $\left\{\theta_{j}^{(i), k}\right\}_{k=1, i=1}^{N p_{0}, 1 / p_{0}} \mapsto \theta_{j}^{(1)}, \ldots, \theta_{j}^{(N)} \sim \pi\left(\cdot \mid F_{j}\right)$

for $i=1, \ldots, N$ do

if $g^{(i)}=g\left(\theta_{j}^{(i)}\right)>b$ do

$N_{F}(j) \leftarrow N_{F}(j)+1$

end if

end for

end while

Output:

- $\hat{p}_{F}^{S S}$, estimate of $p_{F}$ :

$$
\hat{p}_{F}^{S S}=p_{0}^{j} \frac{N_{F}(j)}{N}
$$




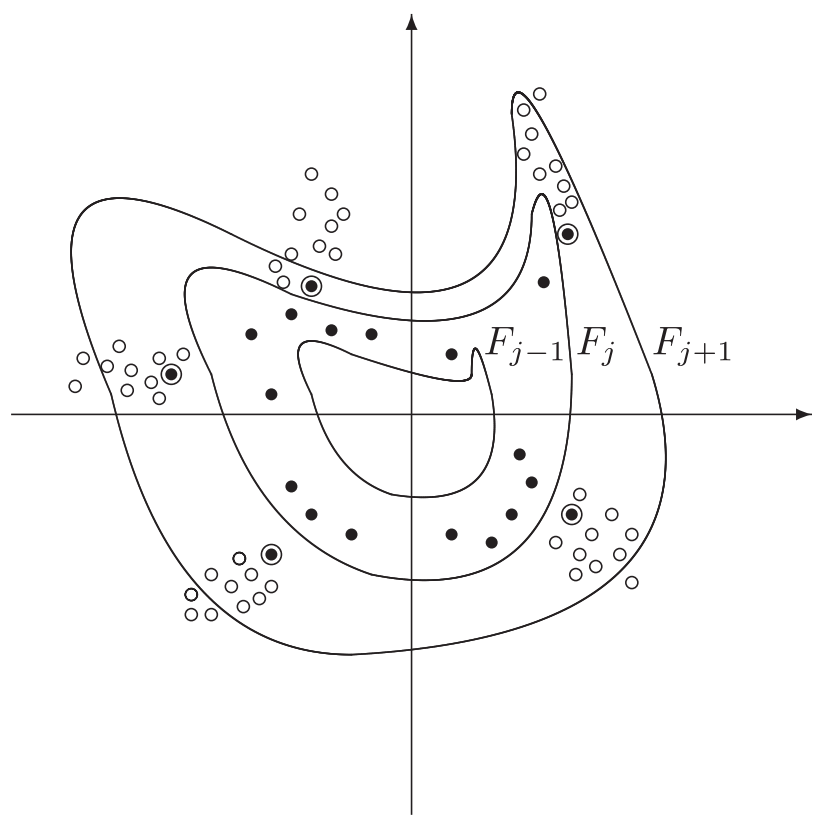

Fig. 2. Subset Simulation algorithm: disks • and circles o represent samples from $\pi\left(\cdot \mid F_{j-1}\right)$ and $\pi\left(\cdot \mid F_{j}\right)$, respectively; circled disks are the Markov chain seeds for $\pi\left(\cdot \mid F_{j}\right)$.

Schematically, the Subset Simulation algorithm is shown in Fig. 2.

The adaptive choice of $b_{j}$-values in (16) guarantees, first, that all seeds $\theta_{j}^{(1), k}$ are distributed according to $\pi\left(\cdot \mid F_{j}\right)$ and, second, that the estimated conditional probability $P\left(F_{j} \mid F_{j-1}\right)$ is equal to $p_{0}$. Here, for convenience, $p_{0}$ is assumed to be chosen such that $N p_{0}$ and $1 / p_{0}$ are positive integers, although this is not strictly necessary. In [2] it is suggested to use $p_{0}=0.1$. The optimal choice of conditional failure probability is discussed in the next section.

Remark 4. Subset Simulation provides an efficient stochastic simulation algorithm for computing failure probabilities for general reliability problems without using any specific information about the dynamic system other than an input-output model. This independence of a system's inherent properties makes Subset Simulation potentially useful for applications in different areas of science and engineering where the notion of "failure" has its own specific meaning, e.g. in Computational Finance to estimate the probability that a stock price will drop below a given threshold within a given period of time, in Computational Biology to estimate the probability of gene mutation, etc.

\section{Tuning of the Modified Metropolis algorithm}

The efficiency and accuracy of Subset Simulation directly depends on the ergodic properties of the Markov chain generated by the Modified Metropolis algorithm; in other words, on how fast the chain explores the parameter space and converges to its stationary distribution. The latter is determined by the choice of one-dimensional proposal distributions $S_{k}$, which makes this choice very important. In spite of this, the choice of proposal PDFs is still largely an art. It was observed in [2] that the efficiency of MMA is not sensitive to the type of the proposal PDFs; however, it depends on their spread (e.g. their variance).

Optimal scaling refers to the need to tune the parameters of the algorithm to make the resulting Markov chain converge to stationarity as fast as possible. The issue of optimal scaling was recognized in the original paper by Metropolis et al. [33]. Gelman et al. [17] were the first authors to obtain theoretical results on the opti- mal scaling of the original Metropolis algorithm. They proved that for optimal sampling from a high-dimensional Gaussian distribution, the Metropolis algorithm should be tuned to accept approximately $23 \%$ of the proposed moves only. Since then many papers have been published on optimal scaling of the original Metropolis algorithm. In this section, in the spirit of [17], we address the following question which is of high practical importance: what is the optimal variance of the univariate Gaussian proposal PDFs for simulating a high-dimensional Gaussian distribution conditional on some specific domain using MMA?

This section is organized as follows: in Section 3.1 we recall the original Metropolis algorithm and provide a brief overview of existing results on its optimal scaling; in Section 3.2 we present a collection of observations on the optimal scaling of the Modified Metropolis algorithm for different numerical examples, and discuss the optimal scaling strategy for MMA when it is employed within Subset Simulation for estimating small failure probabilities.

\subsection{Metropolis algorithm: a brief history of its optimal scaling}

The Metropolis algorithm is the most popular class of MCMC algorithms. Let $\pi$ be the target PDF on $\mathbb{R}^{d} ; \theta^{(n)}$ be the current state of the Markov chain; and $S\left(\cdot \mid \theta^{(n)}\right)$ be a symmetric (i.e. $S(\alpha \mid \beta)=$ $S(\beta \mid \alpha)) d$-variate proposal PDF depending on $\theta^{(n)}$. Then the Metropolis update $\theta^{(n)} \rightarrow \theta^{(n+1)}$ of the Markov chain works as follows: first, simulate a candidate state $\xi \sim S\left(\cdot \mid \theta^{(n)}\right)$; next, compute the acceptance probability $a\left(\xi \mid \theta^{(n)}\right)=\min \left\{1, \pi(\xi) / \pi\left(\theta^{(n)}\right)\right\}$; and, finally, accept $\xi$ as a next state of the Markov chain, i.e. set $\theta^{(n+1)}=\xi$, with probability $a\left(\xi \mid \theta^{(n)}\right)$ or reject $\xi$ by setting $\theta^{(n+1)}=\theta^{(n)}$ with the remaining probability $1-a\left(\xi \mid \theta^{(n)}\right)$. It easy to prove that such updating leaves $\pi$ invariant, i.e. if $\theta^{(n)}$ is distributed according to $\pi$, then so is $\theta^{(n+1)}$. Hence the chain will eventually converge to $\pi$ as its stationary distribution. Practically it means that if we run the Markov chain for a long time, starting from any $\theta^{(1)} \in \mathbb{R}^{d}$, then for large $N$ the distribution of $\theta^{(N)}$ will be approximately $\pi$.

The variance $\sigma^{2}$ of the proposal PDF $S$ turns out to have a significant impact on the speed of convergence of the Markov chain to its stationary distribution. Indeed, if $\sigma^{2}$ is small, then the Markov chain explores its state space very slowly. On the other hand, if $\sigma^{2}$ is large, the probability to accept a new candidate state is very low and this results in a chain remaining still for long periods of time. Since the Metropolis algorithm with extremal values of the variance of the proposal PDF produce a chain that explores its state space slowly, it is natural to expect the existence of an optimal $\sigma^{2}$ for which the convergence speed is maximized.

The importance of optimal scaling was already realized in the landmark paper [33] where the Metropolis algorithm was first introduced. Metropolis et al. developed an algorithm for generating samples from the Boltzmann distribution for solving numerical problems in statistical mechanics. In this work the uniform proposal PDF was used, $S\left(\xi \mid \theta^{(n)}\right)=U_{\left[\theta^{(n)}-\alpha, \theta^{(n)}+\alpha\right]}(\xi)$, and it was noted:

"It may be mentioned in this connection that the maximum displacement $\alpha$ must be chosen with some care; if too large, most moves will be forbidden, and if too small, the configuration will not change enough. In either case it will then take longer to come to equilibrium."

In [18] Hastings generalized the Metropolis algorithm. Namely, he showed that the proposal distribution need not be uniform, and it need not to be symmetric. In the latter case, the acceptance probability must be slightly modified: $a\left(\xi \mid \theta^{(n)}\right)=\min \left\{1, \frac{\pi(\xi) S\left(\theta^{(n)} \mid \xi\right)}{\pi\left(\theta^{(n)}\right) S\left(\xi \mid \theta^{(n)}\right)}\right\}$. The corresponding algorithm is called the Metropolis-Hastings algorithm. Furthermore, Hastings emphasized that the original sampling method has a general nature and can be applied in different circumstances (not only in the framework of statistical 
mechanics) and that Markov chain theory (which is absent in [33]) is a natural language for the algorithm. Among other insights, Hastings made the following useful yet difficult to implement recommendation:

Choose a proposal distribution "so that the sample point in one step may move as large a distance as possible in the sample space, consistent with a low rejection rate."

Historically, the tuning of the proposal's variance was usually performed by trial-and-error, typically using rules of thumb of the following form: select $\sigma^{2}$ such that the corresponding acceptance rate, i.e. the average number of accepted candidate states, is between $30 \%$ and $70 \%$. The rationale behind such rule is that too low an acceptance rate means that the Markov chain has many repeated samples, while too high an acceptance rate indicates that the chain moves very slowly. Although qualitatively correct, these rules suffered from the lack of theoretical justification for the lower and upper bounds for the acceptance rate. The first theoretical result on the optimal scaling of the Metropolis algorithm was obtained by Gelman et al. [17]. It was proved that in order to perform optimally in high dimensional spaces, the algorithm should be tuned to accept as small as $23 \%$ of the proposed moves. This came as an unexpected and counter-intuitive result. Indeed, this states that the Markov chain should stay still about $77 \%$ of the time in order to have the fastest convergence speed. Let us formulate the main result more precisely.

Suppose that all components of $\theta \in \mathbb{R}^{d}$ are i.i.d., i.e. the target distribution $\pi(\theta)$ has the product form, $\pi(\theta)=\prod_{i=1}^{d} f\left(\theta_{i}\right)$, where the one-dimensional density $f$ satisfies certain regularity conditions (namely, $f$ is a $C^{2}$-function and $(\log f)^{\prime}$ is Lipschitz continuous). Then the optimal "random walk" Gaussian proposal PDF $S\left(\xi \mid \theta^{(n)}\right)=$ $\mathcal{N}\left(\xi \mid \theta^{(n)}, \sigma^{2} \rrbracket_{d}\right)$ has the following properties:

1. The optimal standard deviation is $\sigma \approx 2.4 / \sqrt{d I}$, where $I=$ $\mathbb{E}_{f}\left[\left((\log f)^{\prime}\right)^{2}\right]=\int_{-\infty}^{\infty} \frac{\left(f^{\prime}(x)\right)^{2}}{f(x)} d x$ measures the "roughness" of $f$. The smoother the density is, the smaller $I$ is, and, therefore, the larger $\sigma$ is. In particular, for a one-dimensional case $(d=1)$ and standard Gaussian $f(I=1): \sigma \approx 2.4$ (a surprisingly high value!).

2. The acceptance rate of the corresponding Metropolis algorithm is approximately $44 \%$ for $d=1$ and declines to $23 \%$ when $d \rightarrow \infty$. Moreover, the asymptotic optimality of accepting $23 \%$ of proposed moves is approximately true for dimension as low as $d=6$.

This result gives rise to the following useful heuristic strategy, that is easy to implement: tune the proposal variance so that the average acceptance rate is roughly $25 \%$. In spite of the i.i.d. assumption for the target components, this result is believed to be robust and to hold under various perturbations of the target distribution. Being aware of practical difficulties of choosing the optimal $\sigma^{2}$, Gelman et al. provided a very useful observation:

"Interestingly, if one cannot be optimal, it seems better to use too high a value of $\sigma$ than too low."

Remark 5. This observation is consistent with the numerical result obtained recently in [45]: an increased variance of the second stage proposal PDFs improves the performance of the MMHDR algorithm.

Since the pioneering work [17], the problem of optimal scaling has attracted the attention of many researchers and optimal scaling results have been derived for other types of MCMC algorithms. For instance, the Metropolis-adapted Langevin algorithm (MALA) was studied in [36] and it was proved that the asymptotically optimal acceptance rate for MALA is approximately $57 \%$. For a more detailed overview of existing results on the optimal scaling of the Metropolis algorithm see [8] and references cited therein.

\subsection{Optimal scaling of the Modified Metropolis algorithm}

In this section we address two questions: what is the optimal variance $\sigma^{2}$ of the univariate Gaussian proposal PDFs $S_{k}(\cdot \mid \mu)=$ $\mathcal{N}\left(\cdot \mid \mu, \sigma^{2}\right), k=1, \ldots, d$ for simulating a high-dimensional conditional Gaussian distribution $\pi(\cdot \mid F)=\mathcal{N}\left(\cdot \mid 0, \square_{d}\right) I_{F}(\cdot) / P(F)$ using the Modified Metropolis algorithm and what is the optimal scaling strategy for Modified Metropolis when it is employed within Subset Simulation for estimating small failure probabilities?

Let us first define what we mean by "optimal" variance. Let $\theta_{j-1}^{(i), k}$ be the $i$ th sample in the $k$ th Markov chain at simulation level $j-1$. The conditional probability $p_{j}=P\left(F_{j} \mid F_{j-1}\right)$ is then estimated as follows:

$p_{j} \approx \hat{p}_{j}=\frac{1}{N} \sum_{k=1}^{N_{c}} \sum_{i=1}^{N_{s}} I_{F_{j}}\left(\theta_{j-1}^{(i), k}\right), \quad \theta_{j-1}^{(i), k} \sim \pi\left(\cdot \mid F_{j-1}\right)$,

where $N_{c}$ is the number of Markov chains and $N_{s}$ is the total number of samples simulated from each of these chains, $N_{s}=N / N_{c}$, so that the total number of Markov chain samples is $N$. An expression for the coefficient of variation (c.o.v.) of $\hat{p}_{j}$, derived in [2], is given by:

$\delta_{j}=\sqrt{\frac{1-p_{j}}{N p_{j}}\left(1+\gamma_{j}\right)}$,

where

$\gamma_{j}=2 \sum_{i=1}^{N_{s}-1}\left(1-\frac{i}{N_{s}}\right) \frac{R_{j}^{(i)}}{R_{j}^{(0)}}$,

and

$R_{j}^{(i)}=\mathbb{E}\left[I_{F_{j}}\left(\theta_{j-1}^{(1), k}\right) I_{F_{j}}\left(\theta_{j-1}^{(1+i), k}\right)\right]-p_{j}^{2}$

is the autocovariance of the stationary stochastic process $X(i)=$ $I_{F_{j}}\left(\theta_{j-1}^{(i), k}\right)$ at lag $i$. The term $\sqrt{\left(1-p_{j}\right) / N p_{j}}$ in (19) is the c.o.v. of the MCS estimator with $N$ independent samples. The c.o.v. of $\hat{p}_{j}$ can thus be considered as the one in MCS with an effective number of independent samples $N /\left(1+\gamma_{j}\right)$. The efficiency of the estimator using dependent MCMC samples $\left(\gamma_{j}>0\right)$ is therefore reduced compared to the case when the samples are independent $\left(\gamma_{j}=0\right)$. Hence, $\gamma_{j}$ given by (20) can be considered as a measure of correlation between the states of a Markov chain and smaller values of $\gamma_{j}$ imply higher efficiency.

Remark 6. Formula (19) was derived assuming that the Markov chain generated according to MMA is ergodic and that the samples generated by different chains are uncorrelated through the indicator function, i.e. $\mathbb{E}\left[I_{F_{j}}(\theta) I_{F_{j}}\left(\theta^{\prime}\right)\right]-p_{j}^{2}=0$ if $\theta$ and $\theta^{\prime}$ are from different chains. The latter, however, may not be always true, since the seeds for each chain may be dependent. Nevertheless, the expression in (19) provides a useful theoretical description of the c.o.v. of $\hat{p}_{j}$.

Remark 7. The autocovariance sequence $R_{j}^{(i)}, i=0, \ldots, N_{s}-1$, needed for calculation of $\gamma_{j}$, can be estimated using the Markov chain samples at the $(j-1)$ th level by:

$R_{j}^{(i)} \approx \frac{1}{N-i N_{c}} \sum_{k=1}^{N_{c}} \sum_{i^{\prime}=1}^{N_{s}-i} I_{F_{j}}\left(\theta_{j-1}^{\left(i^{\prime}\right), k}\right) I_{F_{j}}\left(\theta_{j-1}^{\left(i^{\prime}+i\right), k}\right)-\hat{p}_{j}^{2}$.

Note that in general, $\gamma_{j}$ depends on the number of samples $N_{s}$ in the Markov chain, the conditional probability $p_{j}$, the intermediate failure domains $F_{j-1}$ and $F_{j}$, and the standard deviation $\sigma_{j}$ of the proposal PDFs $S_{k}(\cdot \mid \mu)=\mathcal{N}\left(\cdot \mid \mu, \sigma_{j}^{2}\right)$. According to the "basic" description of the Subset Simulation algorithm given in Section 2, 
$p_{j}=p_{0}$ for all $j$ and $N_{s}=1 / p_{0}$. The latter, as it has been already mentioned, is not strictly necessary, yet convenient. In this section, the value $p_{0}$ is chosen to be 0.1 , as in the original paper [2]. In this setting, $\gamma_{j}$ depends only on the standard deviation $\sigma_{j}$ and the geometry of $F_{j-1}$ and $F_{j}$. For a given reliability problem (i.e. for a given performance function $g$ that defines domains $F_{j}$ for all $j$ ), $\sigma_{j}^{\text {opt }}$ is said to be the optimal spread of the proposal PDFs at level $j$, if it minimizes the value of $\gamma_{j}$ :

$\sigma_{j}^{\mathrm{opt}}=\arg \min _{\sigma_{j}>0} \gamma_{j}\left(\sigma_{j}\right)$

We will refer to $\gamma_{j}=\gamma_{j}\left(\sigma_{j}\right)$ as $\gamma$-efficiency of the Modified Metropolis algorithm with proposal PDFs $\mathcal{N}\left(\cdot \mid \mu, \sigma_{j}^{2}\right)$ at level $j$.

Consider two examples of the sequence of intermediate failure domains.

Example 1 (Exterior of a ball). Let $\theta=r e \in \mathbb{R}^{d}$, where $e$ is a unit vector and $r=\|\theta\|$. For many reasonable performance functions $g$, if $r$ is large enough, then $\theta \in F=\left\{\theta \in \mathbb{R}^{d}: g(\theta)>b\right\}$, i.e. $\theta$ is a failure point, regardless of $e$. Therefore, an exterior of a ball, $\bar{B}_{r}=$ $\left\{\theta \in \mathbb{R}^{d}:\|\theta\| \geqslant r\right\}$, can serve as an idealized model of many failure domains. Define the intermediate failure domains as follows:

$F_{j}=\bar{B}_{r_{j}}$,

where the radii $r_{j}$ are chosen such that $P\left(F_{j} \mid F_{j-1}\right)=p_{0}$, i.e. $r_{j}^{2}=F_{\chi_{d}^{2}}^{-1}\left(1-p_{0}^{-j}\right)$, where $F_{\chi_{d}^{2}}$ denotes the cumulative distribution function (CDF) of the chi-square distribution with $d$ degrees of freedom. The dimension $d$ is chosen to be $10^{3}$.

Example 2 (Linear case). Consider a linear reliability problem with performance function $g(\theta)=a^{T} \theta+b$, where $a \in \mathbb{R}^{d}$ and $b \in \mathbb{R}$ are fixed coefficients. The corresponding intermediate failure domains $F_{j}$ are half-spaces defined as follows:
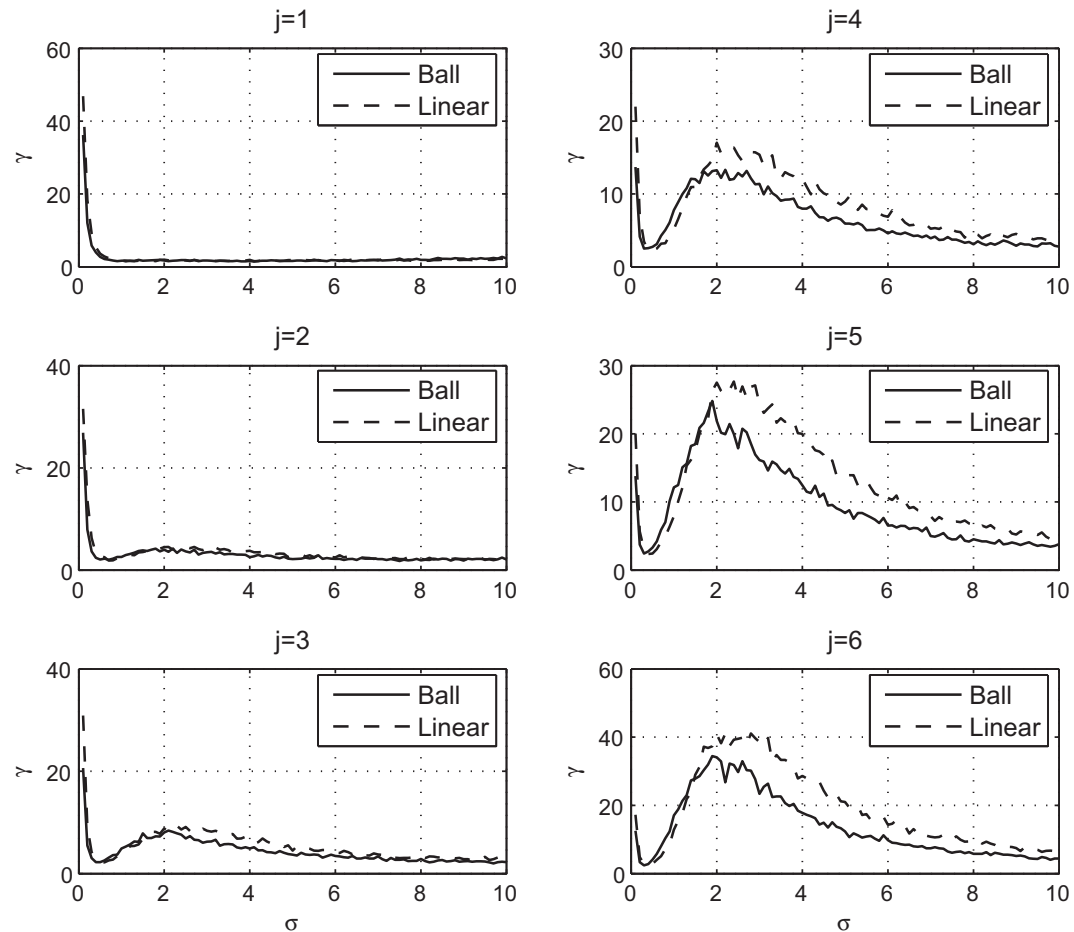

Fig. 3. The $\gamma$-efficiency of the Modified Metropolis algorithm as a function of spread $\sigma$ for simulation levels $j=1, \ldots, 6$. 

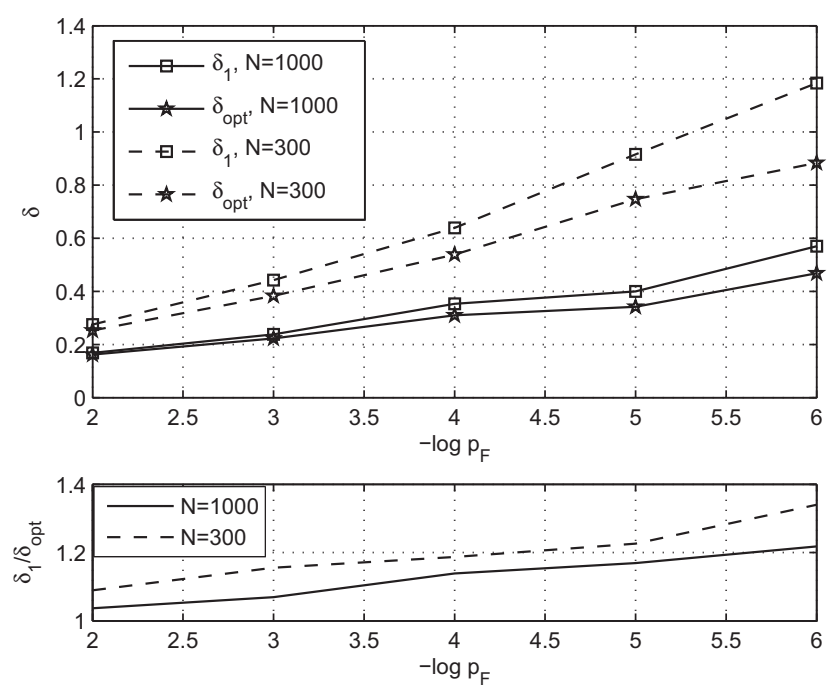

Fig. 4. The c.o.v. of $p_{F}$ estimates obtained by Subset Simulation for Example 1.
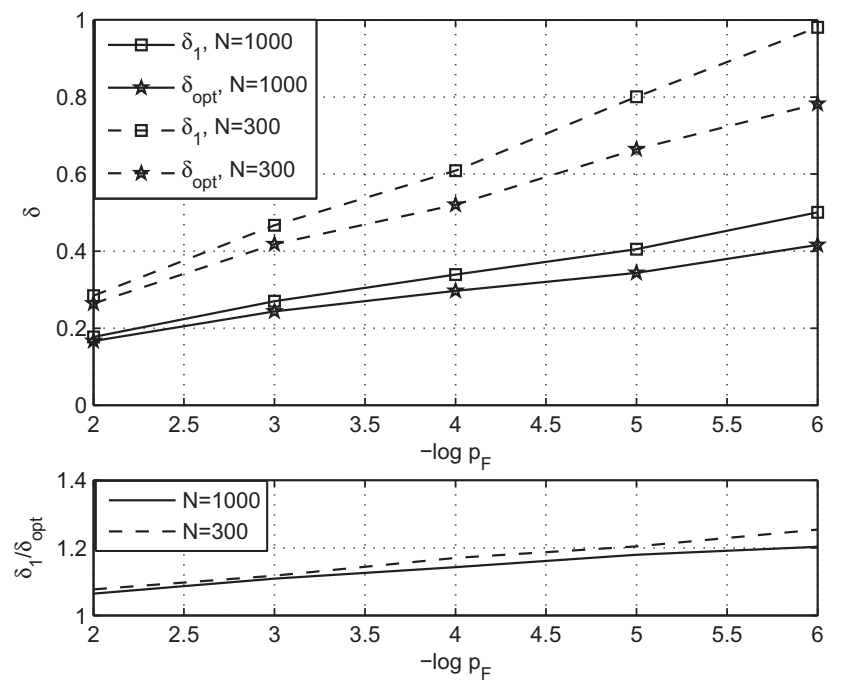

Fig. 5. The c.o.v. of $p_{F}$ estimates obtained by Subset Simulation for Example 2.

The dashed (solid) curves correspond to the case when $N=300$ $(N=1000)$ samples are used per each intermediate failure region. For estimation of each value of the failure probability, two different MMAs are used within SS: the optimal algorithm with $\sigma_{j}=\sigma_{j}^{\text {opt }}$ (marked with stars); and the reference algorithm with $\sigma_{j}=1$ (marked with squares). The corresponding c.o.v.'s are denoted by $\delta_{\text {opt }}$ and $\delta_{1}$, respectively. From Figs. 4 and 5 it follows that the smaller $p_{F}$, the more important to scale MMA optimally. When $p_{F}=10^{-6}$, the optimal c.o.v. $\delta_{\text {opt }}$ is approximately $80 \%$ of the reference c.o.v. $\delta_{1}$ for both examples, when $N=1000$.

Despite its obvious usefulness, the optimal scaling of the Modified Metropolis algorithm is difficult to achieve in practice. First, as shown in Table 1 , the values of the optimal spread $\sigma_{j}^{\text {opt }}$ are different for different reliability problems. Second, even for a given reliability problem, to find $\sigma_{j}^{\text {opt }}$ is computationally expensive because of property (ii) of $\gamma_{j}$; and our simulation results show that the qualitative properties (i) and (ii) generally hold for different reliability problems, not only for Examples 1 and 2. Therefore, we look for heuristic to choose $\sigma_{j}$ that is easy to implement and yet gives near optimal behavior.

It has been recognized for a long time that, when using an MCMC algorithm, it is useful to monitor its acceptance rate. Both
Table 2

Approximate values of the acceptance rates $\rho_{j}(1)$ and $\rho_{j}^{\text {opt }}$ that correspond to the reference value $\sigma_{j}=1$ and the optimal spread $\sigma_{j}=\sigma_{j}^{\text {opt }}$, respectively.

\begin{tabular}{lllllll}
\hline & \multicolumn{7}{l}{ Simulation level $j$} \\
\cline { 2 - 7 } & 1 & 2 & 3 & 4 & 5 & 6 \\
\hline Example 1, $\rho_{j}(1)$ & $49 \%$ & $30 \%$ & $20 \%$ & $13 \%$ & $9 \%$ & $6 \%$ \\
Example 1, $\rho_{j}^{\text {opt }}$ & $51 \%$ & $39 \%$ & $47 \%$ & $50 \%$ & $44 \%$ & $40 \%$ \\
Example 2, $\rho_{j}(1)$ & $53 \%$ & $35 \%$ & $23 \%$ & $16 \%$ & $11 \%$ & $8 \%$ \\
Example 2, $\rho_{j}^{\text {opt }}$ & $52 \%$ & $41 \%$ & $40 \%$ & $49 \%$ & $43 \%$ & $37 \%$ \\
\hline
\end{tabular}

$\gamma$-efficiency $\gamma_{j}$ and the acceptance rate $\rho_{j}$ at level $j$ depend on $\sigma_{j}$. For Examples 1 and 2, the approximate values of the acceptance rate that corresponds to the reference value $\sigma_{j}=1$ and the optimal spread $\sigma_{j}=\sigma_{j}^{\mathrm{opt}}$ are given in Table $2 ; \gamma_{j}$ as a function of $\rho_{j}$ is plotted in Fig. 6 , for simulation levels $j=1, \ldots, 6$. A key observation is that, contrary to (ii), $\gamma_{j}$ is very flat around the optimal acceptance rate $\rho_{j}^{\text {opt }}$, which is defined as the acceptance rate that corresponds to the optimal spread, i.e. $\rho_{j}^{\text {opt }}=\rho_{j}\left(\sigma_{j}^{\text {opt }}\right)$. Furthermore, according to our simulation results this behavior is typical, and not specific just for the considered examples. This observation gives rise to the following heuristic scaling strategy:

At simulation level $j \geqslant 1$ select $\sigma_{j}$ such that the corresponding acceptance rate $\rho_{j}$ is between $30 \%$ and $50 \%$.

This strategy is easy to implement in the context of Subset Simulation. At each simulation level $j, N_{c}$ Markov chains are generated. Suppose, we do not know the optimal spread $\sigma_{j}^{\text {opt }}$ for our problem. We start with a reference value, say $\sigma_{j}^{1: n}=1$, for the first $n$ chains. Based only on these $n$ chains, we calculate the corresponding acceptance rate $\rho_{j}^{1: n}$. If $\rho_{j}^{1: n}$ is too low (i.e. it is smaller than 30\%) we decrease the spread and use $\sigma_{j}^{n+1: 2 n}<\sigma_{j}^{1: n}$ for the next $n$ chains. If $\rho_{j}^{1: n}$ is too large (i.e. it is larger than 50\%) we increase the spread and use $\sigma_{j}^{n+1: 2 n}>\sigma_{j}^{1: n}$ for the next $n$ chains. We proceed like this until all $N_{c}$ Markov chains have been generated. Note that according to this procedure, $\sigma_{j}$ is kept constant within a single chain and it is changed only between chains. Hence the Markovian property is not destroyed. The described strategy guaranties that the corresponding scaling on the Modified Metropolis algorithm is nearly optimal.

\section{Optimal choice of conditional failure probability $p_{0}$}

The parameter $p_{0}$ governs how many intermediate failure domains $F_{j}$ are needed to reach the target failure domain $F$, which in turn affects the efficiency of Subset Simulation. A very small value of the conditional failure probability means that fewer intermediate levels are needed to reach $F$ but it results in a very large number of samples $N$ needed at each level for accurate estimation of the small conditional probabilities $p_{j}=P\left(F_{j} \mid F_{j-1}\right)$. In the extreme case when $p_{0} \leqslant p_{F}$, Subset Simulation reduces to the standard Monte Carlo simulation. On the other hand, increasing the value of $p_{0}$ will mean fewer samples are needed for accurate estimation at each level but it will increase the number of intermediate conditional levels $m$. In this section we provide a theoretical basis for the optimal value of the conditional failure probability.

We wish to choose the value $p_{0}$ such that the coefficient of variation (c.o.v.) of the failure probability estimator $\hat{p}_{F}^{S S}$ is as small as possible, for the same total number of samples. In [2], an analysis of the statistical properties of the Subset Simulation estimator is given. If the conditional failure domains are chosen so that the corresponding estimates of the conditional probabilities are all equal to $p_{0}$ and the same number of samples $N$ is used in the simulation at each conditional level, then the c.o.v. of the estimator $\hat{p}_{F}^{S S}$ for a 

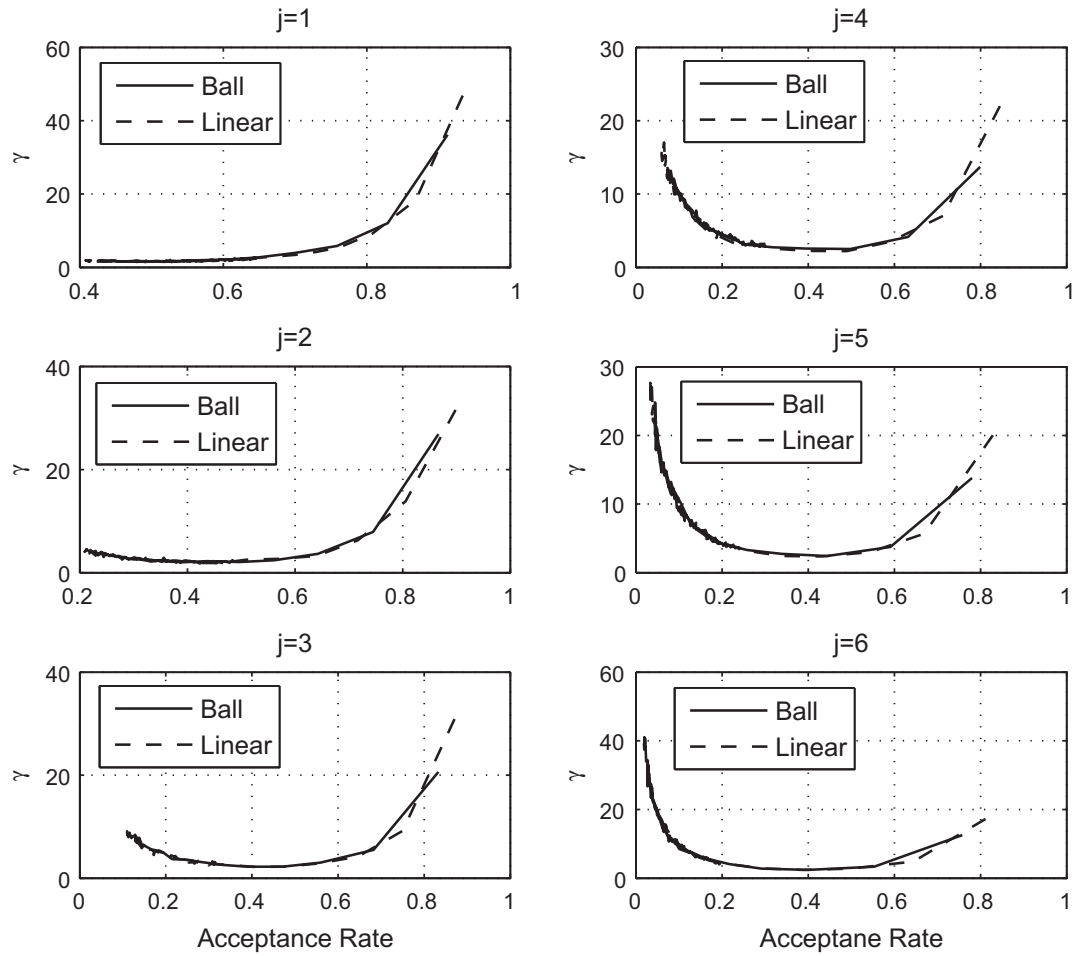

Fig. 6. The $\gamma$-efficiency of the Modified Metropolis algorithm as a function of the acceptance rate for simulation levels $j=1, \ldots, 6$.

failure probability $p_{F}=p_{0}^{m}$ (requiring $m$ conditional levels) is approximated by

$\delta^{2} \approx \frac{m\left(1-p_{0}\right)}{N p_{0}}(1+\bar{\gamma})$

where $\bar{\gamma}$ is the average correlation factor over all levels (assumed to be insensitive to $p_{0}$ ) that reflects the correlation among the MCMC samples in each level and depends on the choice of the spread of the proposal PDFs. Since the total number of samples $N_{T}=m N$ and the number of conditional levels $m=\log p_{F} / \log p_{0}$, (26) can be rewritten as follows:

$\delta^{2} \approx \frac{1-p_{0}}{p_{0}\left(\log p_{0}\right)^{2}} \times \frac{\left(\log p_{F}\right)^{2}}{N_{T}}(1+\bar{\gamma})$.

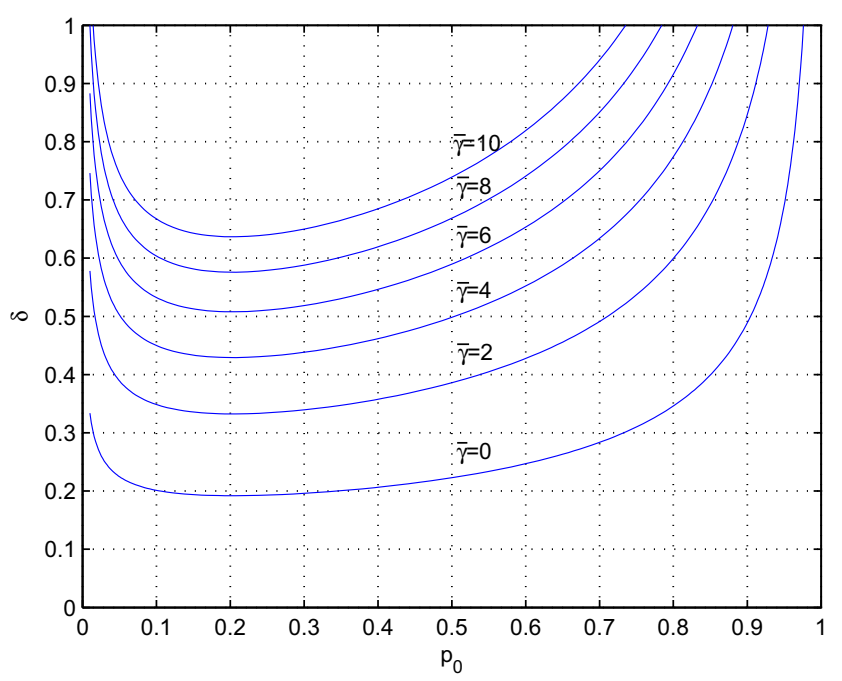

Fig. 7. Variation of $\delta$ as a function of $p_{0}$ according to (27) for $p_{F}=10^{-3}, N_{T}=2000$, and $\bar{\gamma}=0,2,4,6,8,10$.
Note that for given target failure probability $p_{F}$ and the total number of samples $N_{T}$, the second factor in (27) does not depend on $p_{0}$. Thus, minimizing the first factor to minimize the c.o.v. $\delta$ yields the optimal value as $p_{0}^{\text {opt }} \approx 0.2$. Fig. 7 shows the variation of $\delta$ as a function of $p_{0}$ according to (27) for $p_{F}=10^{-3}, N_{T}=2000$, and $\bar{\gamma}=0,2,4,6$, 8,10 . This figure indicates that $\delta$ is relatively insensitive to $p_{0}$ around its optimal value. Note that the shape of the trend is invariant with respect to $p_{F}, N_{T}$ and $\bar{\gamma}$ because their effects are multiplicative. The figure shows that choosing $0.1 \leqslant p_{0} \leqslant 0.3$ will practically lead to similar efficiency and it is not necessary to fine tune the value of the conditional failure probability $p_{0}$ as long as Subset Simulation is implemented properly.

\section{Bayesian post-processor for Subset Simulation}

In this section we develop a Bayesian post-processor SS+ for the original Subset Simulation algorithm described in Section 2, that provides more information about the value of $p_{F}$ than a single point estimate.

Recall that in SS the failure probability $p_{F}$ is represented as a product of conditional probabilities $p_{j}=P\left(F_{j} \mid F_{j-1}\right)$, each of which is estimated using (6) for $j=1$ and (12) for $j=2, \ldots, m$. Let $n_{j}$ denote the number of samples $\theta_{j-1}^{(1)}, \ldots, \theta_{j-1}^{(N)}$ that belong to subset $F_{j}$. The estimate for probability $p_{j}$ is then:

$\hat{p}_{j}=\frac{n_{j}}{N}$

and the estimate for the failure probability defined by (1) is:

$\hat{p}_{F}^{S S}=\prod_{j=1}^{m} \hat{p}_{j}=\prod_{j=1}^{m} \frac{n_{j}}{N}$.

In order to construct a Bayesian post-processor for SS, we have to replace the frequentist estimates (28) in (29) by their Bayesian analogs. In other words, we have to treat all $p_{1}, \ldots, p_{m}$ and $p_{F}$ as stochastic variables and, following the Bayesian approach, proceed as follows: 
1. Specify prior PDFs $p\left(p_{j}\right)$ for all $p_{j}=P\left(F_{j} \mid F_{j-1}\right), j=1, \ldots, m$;

2. Update each prior PDF, using new data $\mathcal{D}_{j-1}=\left\{\theta_{j-1}^{(1)}, \ldots, \theta_{j-1}^{(N)} \sim\right.$ $\left.\pi\left(\cdot \mid F_{j-1}\right)\right\}$, i.e. find the posterior PDFs $p\left(p_{j} \mid \mathcal{D}_{j-1}\right)$ via Bayes' theorem;

3. Obtain the posterior PDF $p\left(p_{F} \mid \cup_{j=0}^{m-1} \mathcal{D}_{j}\right)$ of $p_{F}=\prod_{j=1}^{m} p_{j}$ from $p\left(p_{1} \mid \mathcal{D}_{0}\right), \ldots, p\left(p_{m} \mid \mathcal{D}_{m-1}\right)$.

Remark 8. The term "stochastic variable" is used rather then "random variable" to emphasize that it is a variable whose value is uncertain, not random, based on the limited information that we have available, and for which a probability model is chosen to describe the relative plausibility of each of its possible values $[7,21]$. The failure probability $p_{F}$ is a constant given by (1) which lies in $[0,1]$ but its exact value is unknown because the integral cannot be evaluated exactly, and so we quantify the plausibility of its values based on the samples that probe the performance function $g$.

To choose the prior distribution for each $p_{j}$, we use the Principle of Maximum Entropy (PME), introduced by Jaynes [19]. The PME postulates that, subject to specified constraints, the prior PDF $p$ which should be taken to represent the prior state of knowledge is the one that gives the largest measure of uncertainty, i.e. maximizes Shannon's entropy which for a continuous variable is given by $H(p)=-\int_{-\infty}^{\infty} p(x) \log p(x) d x$. Since the set of all possible values for each stochastic variable $p_{j}$ is the unit interval, we impose this as the only constraint for $p\left(p_{j}\right)$, i.e. $\operatorname{supp} p\left(p_{j}\right)=[0,1]$. It is well known that the uniform distribution is the maximum entropy distribution among all continuous distributions on $[0,1]$, so

$p\left(p_{j}\right)=1, \quad 0 \leqslant p_{j} \leqslant 1$.

Remark 9. We could choose a more informative prior PDF, perhaps based on previous experience with the failure probabilities for similar systems. If the amount of data is large (i.e. $N$ is large), however, then the effect of the prior PDF on the posterior PDF will be negligible if the likelihood function has a unique global maximum. This phenomenon is usually referred to in the literature as the "stability" or "robustness" of Bayesian estimation.

Since initial samples $\theta_{0}^{(1)}, \ldots, \theta_{0}^{(N)}$ are i.i.d. according to $\pi$, the sequence of zeros and ones, $I_{F_{1}}\left(\theta_{0}^{(1)}\right), \ldots, I_{F_{1}}\left(\theta_{0}^{(N)}\right)$, can be considered as Bernoulli trials and, therefore, the likelihood function $p\left(\mathcal{D}_{0} \mid p_{1}\right)$ is a binomial distribution where $\mathcal{D}_{0}$ consists of the number of $F_{1}$-failure samples $n_{1}=\sum_{k=1}^{N} I_{F_{1}}\left(\theta_{0}^{(k)}\right)$ and the total number of samples is $N$. Hence, the posterior distribution of $p_{1}$ is the beta distribution $\mathcal{B} e\left(n_{1}+1, N-n_{1}+1\right)$ (e.g. [16]) with parameters $\left(n_{1}+1\right)$ and $\left(N-n_{1}+1\right)$, i.e.

$p\left(p_{1} \mid \mathcal{D}_{0}\right)=\frac{p_{1}^{n_{1}}\left(1-p_{1}\right)^{N-n_{1}}}{\mathrm{~B}\left(n_{1}+1, N-n_{1}+1\right)}$,

which is actually the original Bayes' result [6]. The beta function B in (31) is a normalizing constant. If $j \geqslant 2$, all MCMC samples $\theta_{j-1}^{(1)}, \ldots, \theta_{j-1}^{(N)}$ are distributed according to $\pi\left(\cdot \mid F_{j-1}\right)$, however, they are not independent. Nevertheless, analogously to the frequentist case, where we used these samples for statistical averaging (12) as if they were i.i.d., we can use an expression similar to (31) as a good approximation for the posterior PDF $p\left(p_{j} \mid \mathcal{D}_{j-1}\right)$ for $j \geqslant 2$, so:

$p\left(p_{j} \mid \mathcal{D}_{j-1}\right) \approx \frac{p_{j}^{n_{j}}\left(1-p_{j}\right)^{N-n_{j}}}{\mathrm{~B}\left(n_{j}+1, N-n_{j}+1\right)}, j \geqslant 1$,

where $n_{j}=\sum_{k=1}^{N} I_{F_{j}}\left(\theta_{j-1}^{(k)}\right)$ is the number of $F_{j}$-failure samples. Note that in Subset Simulation the MCMC samples $\theta_{j-1}^{(1)}, \ldots, \theta_{j-1}^{(N)}$ consist of the states of multiple Markov chains with different initial seeds obtained from previous conditional levels. This makes the approxi- mation (32) more accurate in comparison with the case of a single chain.

Remark 10. It is important to highlight that using the r.h.s of (32) as the posterior PDF $p\left(p_{j} \mid \mathcal{D}_{j-1}\right)$ is equivalent to considering samples $\theta_{j-1}^{(1)}, \ldots, \theta_{j-1}^{(N)}$ as independent. This probability model ignores information that the samples are generated by an MCMC algorithm, just as it ignores the fact that the random number generator used to generate these samples is, in fact, a completely deterministic procedure. One corollary of this neglected information is that generally $\delta_{p\left(p_{F}\right)}<\delta_{\hat{p}_{F}^{s s}}$, where $\delta_{p\left(p_{F}\right)}$ is the c.o.v. of the posterior PDF of $p_{F}$ and $\delta_{\hat{p}_{F}^{s S}}$ is the c.o.v. of the original SS estimator $\hat{p}_{F}^{S S}$ (see also the numerical examples in Section 6). Notice, however, that the two c.o.v.s are fundamentally different: $\delta_{p\left(p_{F}\right)}$ is defined based on samples generated from a single run of SS, while the frequentist c.o.v. $\delta_{\hat{p}_{F}^{s s}}$ is defined based on repeated runs of SS (an infinite number of them!).

The last step is to find the PDF of the product of stochastic variables $p_{F}=\prod_{j=1}^{m} p_{j}$, given the distributions of all factors $p_{j}$ by (32).

Remark 11. Products of random (or stochastic) variables play a central role in many different fields such as physics (interactive particle systems), number theory (asymptotic properties of arithmetical functions), statistics (asymptotic distributions of order statistics), etc. The theory of products of random variables is well covered in [15].

In general, to find the distribution of a product of stochastic variables is a non-trivial task. A well-known result is Rohatgi's formula [37]: if $X_{1}$ and $X_{2}$ are continuous stochastic variables with joint $\operatorname{PDF} f_{X_{1}, X_{2}}$, then the PDF of $Y=X_{1} X_{2}$ is

$f_{Y}(y)=\int_{-\infty}^{+\infty} f_{X_{1}, X_{2}}\left(x, \frac{y}{x}\right) \frac{1}{|x|} d x$

This result is straightforward to derive but it is difficult to implement, especially when the number of stochastic variables is more than two. In the special case of a product of independent beta variables, Tang and Gupta [40] derived an exact representation for the PDF and provided a recursive formula for computing the coefficients of this representation.

Theorem 1 [40]. Let $X_{1}, \ldots, X_{m}$ be independent beta variables, $X_{j} \sim \mathcal{B e}\left(a_{j}, b_{j}\right)$, and $Y=X_{1} X_{2} \cdots X_{m}$, then the probability density function of $Y$ can be written as follows:

$$
\begin{aligned}
f_{Y}(y)= & \left(\prod_{j=1}^{m} \frac{\Gamma\left(a_{j}+b_{j}\right)}{\Gamma\left(a_{j}\right)}\right) y^{a_{m}-1}(1-y)^{\sum_{j=1}^{m} b_{j}-1} \\
& \cdot \sum_{r=0}^{\infty} \sigma_{r}^{(m)}(1-y)^{r}, \quad 0<y<1,
\end{aligned}
$$

where $\Gamma$ is the gamma function and coefficients $\sigma_{r}^{(m)}$ are defined by the following recurrence relation:

$$
\begin{aligned}
& \sigma_{r}^{(k)}=\frac{\Gamma\left(\sum_{j=1}^{k-1} b_{j}+r\right)}{\Gamma\left(\sum_{j=1}^{k} b_{j}+r\right)} \sum_{s=0}^{r} \frac{\left(a_{k}+b_{k}-a_{k-1}\right)_{s}}{s !} \sigma_{r-s}^{(k-1)}, \\
& r=0,1, \ldots, k=2, \ldots, m
\end{aligned}
$$

with initial values

$$
\sigma_{0}^{(1)}=\frac{1}{\Gamma\left(b_{1}\right)}, \quad \sigma_{r}^{(1)}=0 \text { for } r \geqslant 1
$$

Here, for any real number $\alpha \in \mathbb{R},(\alpha)_{s}=\alpha(\alpha+1) \cdots(\alpha+s-1)=\frac{\Gamma(\alpha+s)}{\Gamma(\alpha)}$.

We can obtain the posterior PDF of stochastic variable $p_{F}$ by applying this theorem to $p_{F}=\prod_{j=1}^{m} p_{j}$, where $p_{j} \sim \mathcal{B} e\left(n_{j}+1, N-\right.$ $\left.n_{j}+1\right)$. 
Let $p^{S S+}\left(p_{F} \mid \cup_{j=0}^{m-1} \mathcal{D}_{j}\right)$ denote the right-hand side of (34) with $a_{j}=n_{j}+1$ and $b_{j}=N-n_{j}+1$, and $\hat{p}_{M A P}^{S S+}$ be the maximum a posteriori (MAP) estimate, i.e. the mode of $p^{S S+}$ :

$\hat{p}_{M A P}^{S S+}=\arg \max _{p_{F} \in[0,1]} p^{S S+}\left(p_{F} \mid \cup_{j=0}^{m-1} \mathcal{D}_{j}\right)$.

Since the mode of a product of independent stochastic variables is equal to the product of the modes, and the mode of the beta variable $X \sim \mathcal{B e}(a, b)$ is $(a-1) /(a+b-2)$, we have:

$$
\begin{aligned}
\hat{p}_{M A P}^{S S+} & =\operatorname{mode}\left(p^{S S+}\left(p_{F} \mid \cup_{j=0}^{m-1} \mathcal{D}_{j}\right)\right)=\prod_{j=1}^{m} \operatorname{mode}\left(p\left(p_{j} \mid \mathcal{D}_{j-1}\right)\right) \\
& =\prod_{j=1}^{m} \frac{n_{j}}{N}=\hat{p}_{F}^{S S} .
\end{aligned}
$$

Thus, the original estimate $\hat{p}_{F}^{S S}$ of failure probability obtained in the original Subset Simulation algorithm is just the MAP estimate $\hat{p}_{M A P}^{S S+}$ corresponding to the PDF $p^{S S+}$. This is a consequence of the choice of a uniform prior.

Although (34) provides an exact expression for the PDF of $Y$, it contains an infinite sum that must be replaced by a truncated finite sum in actual computations. This means that in applications one has to use an approximation of the posterior PDF $p^{S S+}$ based on (34). An alternative approach is to approximate the distribution of the product $Y=\prod_{j=1}^{m} X_{j}$ by a single beta variable $\widetilde{Y} \sim \mathcal{B} e(a, b)$, where the parameters $a$ and $b$ are chosen so that $\mathbb{E}[\widetilde{Y}]=\mathbb{E}[Y]$ and $\mathbb{E}\left[\widetilde{Y}^{k}\right]$ is as close to $\mathbb{E}\left[Y^{k}\right]$ as possible for $2 \leqslant k \leqslant K$, for some fixed $K$. This idea was first proposed in [42]. In general, the product of beta variables does not follow the beta distribution, nevertheless, it was shown in [13] that the product can be accurately approximated by a beta variable even in the case of $K=2$.

Theorem 2 [13]. Let $X_{1}, \ldots, X_{m}$ be independent beta variables, $X_{j} \sim \mathcal{B e}\left(a_{j}, b_{j}\right)$, and $Y=X_{1} X_{2} \cdots X_{m}$, then $Y$ is approximately distributed as $\widetilde{Y} \sim \mathcal{B e}(a, b)$, where

$a=\mu_{1} \frac{\mu_{1}-\mu_{2}}{\mu_{2}-\mu_{1}^{2}}, \quad b=\left(1-\mu_{1}\right) \frac{\mu_{1}-\mu_{2}}{\mu_{2}-\mu_{1}^{2}}$,

and

$\mu_{1}=\mathbb{E}[Y]=\prod_{j=1}^{m} \frac{a_{j}}{a_{j}+b_{j}}, \quad \mu_{2}=\mathbb{E}\left[Y^{2}\right]=\prod_{j=1}^{m} \frac{a_{j}\left(a_{j}+1\right)}{\left(a_{j}+b_{j}\right)\left(a_{j}+b_{j}+1\right)}$.

It is easy to check that if $\widetilde{Y} \sim \mathcal{B} e(a, b)$ with $a$ and $b$ given by (39), then the first two moments of stochastic variables $Y$ and $\widetilde{Y}$ coincide, i.e. $\mathbb{E}[\widetilde{Y}]=\mathbb{E}[Y]$ and $\mathbb{E}\left[\widetilde{Y}^{2}\right]=\mathbb{E}\left[Y^{2}\right]$. The accuracy of the approximation $Y \dot{\sim} \mathcal{B e}(a, b)$ is discussed in [13].

Using Theorem 2, we can therefore approximate the posterior distribution $p^{S S+}$ of stochastic variable $p_{F}$ by the beta distribution as follows:

$$
\begin{aligned}
p^{S S+}\left(p_{F} \mid \cup_{j=0}^{m-1} \mathcal{D}_{j}\right) & \approx \tilde{p}^{S S+}\left(p_{F} \mid \cup_{j=0}^{m-1} \mathcal{D}_{j}\right) \\
& =\mathcal{B} e\left(p_{F} \mid a, b\right), \quad \text { i.e. } p_{F} \dot{\sim} \mathcal{B} e(a, b),
\end{aligned}
$$

where

$a=\frac{\prod_{j=1}^{m} \frac{n_{j}+1}{N+2}\left(1-\prod_{j=1}^{m} \frac{n_{j}+2}{N+3}\right)}{\prod_{j=1}^{m} \frac{n_{j}+2}{N+3}-\prod_{j=1}^{m} \frac{n_{j}+1}{N+2}}, \quad b=\frac{\left(1-\prod_{j=1}^{m} \frac{n_{j}+1}{N+2}\right)\left(1-\prod_{j=1}^{m} \frac{n_{j}+2}{N+3}\right)}{\prod_{j=1}^{m} \frac{n_{j}+2}{N+3}-\prod_{j=1}^{m} \frac{n_{j}+1}{N+2}}$.

Since the first two moments of $p^{S S+}$ and $\tilde{p}^{S S+}$ are equal (this also means the c.o.v. of $p^{S S+}$ and $\tilde{p}^{S S+}$ are equal), we have:

$$
\begin{aligned}
& \mathbb{E}_{\tilde{p} s{ }^{S+}}\left[p_{F}\right]=\mathbb{E}_{p S S+}\left[p_{F}\right]=\prod_{j=1}^{m} \mathbb{E}_{p\left(p_{j} \mid \mathcal{D}_{j-1}\right)}\left[p_{j}\right]=\prod_{j=1}^{m} \frac{n_{j}+1}{N+2}, \\
& \mathbb{E}_{\hat{p}^{S S+}}\left[p_{F}^{2}\right]=\mathbb{E}_{p S S+}\left[p_{F}^{2}\right]=\prod_{j=1}^{m} \mathbb{E}_{p\left(p_{j} \mid \mathcal{D}_{j-1}\right)}\left[p_{j}^{2}\right]=\prod_{j=1}^{m} \frac{\left(n_{j}+1\right)\left(n_{j}+2\right)}{(N+2)(N+3)}
\end{aligned}
$$

Notice that

$\lim _{N \rightarrow \infty} \mathbb{E}_{\tilde{p}^{S S+}}\left[p_{F}\right]=\lim _{N \rightarrow \infty} \hat{p}_{F}^{S S}$, and $\mathbb{E}_{\tilde{p}^{S S_{+}}}\left[p_{F}\right] \approx \hat{p}_{F}^{S S}$, when $N$ is large

so, the mean value of the approximation $\tilde{p}^{S S+}$ to the posterior PDF $p^{S S+}$ is accurately approximated by the original estimate $\hat{p}_{F}^{S S}$ of the failure probability $p_{F}$.

Let us now summarize the Bayesian post-processor of Subset Simulation. From the algorithmic point of view, SS+ differs from SS only in the produced output. Instead of a single real number as an estimate of $p_{F}$, SS+ produces the posterior PDF $p^{S S+}\left(p_{F} \mid \cup_{j=0}^{m-1} \mathcal{D}_{j}\right)$ of the failure probability, which takes into account both prior information and the sampled data $\cup_{j=0}^{m-1} \mathcal{D}_{j}$ generated by SS, while its approximation $\tilde{p}^{S S+}\left(p_{F} \mid \cup_{j=0}^{m-1} \mathcal{D}_{j}\right)$ is more convenient for further computations. The posterior PDF $p^{S S+}$ and its approximation $\tilde{p}^{S S+}$ are given by (34) and (41), respectively, where

$n_{j}= \begin{cases}p_{0} N, & \text { if } j<m \\ N_{F}, & \text { if } j=m,\end{cases}$

and $m$ is the total number of intermediate levels in the run of the algorithm. The relationship between SS and SS+ is given by (38) and (44). Namely, the original estimate $\hat{p}_{F}^{S S}$ of the failure probability based on the samples produced by the Subset Simulation algorithm coincides with the MAP estimate corresponding to $p^{S S+}$, and it also accurately approximates the mean of $\tilde{p}^{S S+}$. Also, the c.o.v. ofp ${ }^{S S+}$ and $\tilde{p}^{S S+}$ coincide and can be computed using the first two moments in (43).

Remark 12. Note that to incorporate the uncertainty in the value of $p_{F}$, one can use the full PDF $\tilde{p}^{S S+}$ for life-cycle cost analyses, decision making under risk, and so on, rather than just using a point estimate of $p_{F}$. For instance, a performance loss function $\mathcal{L}$ often depends on the failure probability. In this case one can calculate an expected loss given by the following integral:

$\mathbb{E}\left[\mathcal{L}\left(p_{F}\right)\right]=\int_{0}^{1} \mathcal{L}\left(p_{F}\right) \tilde{p}^{S S+}\left(p_{F}\right) d p_{F}$,

which takes into account the uncertainty in the value of the failure probability.

Remark 13. We note that a Bayesian post-processor for Monte Carlo evaluation of the integral (1), denoted as MC+, can be obtained as a special case of SS+. The posterior PDF for the failure probability $p_{F}$ based on $N$ i.i.d. samples $\mathcal{D}=\left\{\theta^{(1)}, \ldots, \theta^{(\mathrm{N})}\right\}$ from $\pi(\cdot)$ is given by

$p^{M C+}\left(p_{F} \mid \mathcal{D}\right)=\operatorname{Be}\left(p_{F} \mid n+1, N-n+1\right)$,

where $n=\sum_{k=1}^{N} I_{F}\left(\theta^{(k)}\right)$ is the number of failure samples.

\section{Illustrative examples}

To demonstrate the Bayesian post-processor of Subset Simulation, we consider its application to two different reliability problems: a linear reliability problem and reliability analysis of an elasto-plastic structure subjected to strong seismic ground motion. 


\subsection{Linear reliability problem}

As a first example, consider a linear failure domain. Let $d=10^{3}$ be the dimension of the linear problem and suppose $p_{F}=10^{-3}$ is the exact failure probability. The failure domain $F$ is defined as

$F=\left\{\theta \in \mathbb{R}^{d}:\langle\theta, e\rangle \geqslant \beta\right\}$,

where $e$ is a unit vector and $\beta=\Phi^{-1}\left(1-p_{F}\right) \approx 3.09$ is the reliability index. This example is one where FORM $[32,27]$ gives the exact failure probability in terms of $\beta$. Note that $\theta^{*}=\beta e$ is the design point of the failure domain $F[26,32]$. The failure probability estimate $\hat{p}_{F}^{S S}$ obtained by SS and the approximation of the posterior PDF $\tilde{p}^{S S+}$ obtained by SS+ are given in Fig. 8 based on a number of samples $N=10^{3}$ at each level ( $m=3$ levels were needed). Observe that $\tilde{p}^{S S+}$ is quite narrowly focused (with the mean $\mu_{\hat{p} s S_{+}}=1.064 \times 10^{-3}$ and the c.o.v. $\delta_{\tilde{p}^{s S+}}=0.16$ ) around $\hat{p}_{F}^{S S}=1.057 \times 10^{-3}$, which is very close to the exact value. Note that the frequentist c.o.v. of the original SS estimator $\hat{p}_{F}^{S S}$ is $\delta_{\hat{p}_{F}^{s S}}=0.28$ (based on 50 independent runs of the algorithm).

\subsection{Elasto-plastic structure subjected to ground motion}

This example of a non-linear system is taken from [1]. Consider a structure that is modeled as a 2D six-story moment-resisting steel frame with two-node beam elements connecting the joints of the frame. The floors are assumed to be rigid in-plane and the joints are assumed to be rigid-plastic. The yield strength is assumed to be $317 \mathrm{MPa}$ for all members. Under service load condition, the floors and the roof are subjected to a uniformly-distributed static span load of $24.7 \mathrm{kN} / \mathrm{m}$ and $13.2 \mathrm{kN} / \mathrm{m}$, respectively. For the horizonal motion of the structure, masses are lumped at the floor levels, which include contributions from live loads and the dead loads from the floors and the frame members. The natural frequencies of the first two modes of vibration are computed to be $0.61 \mathrm{~Hz}$ and $1.71 \mathrm{~Hz}$. Rayleigh damping is assumed so that the first two modes have $2 \%$ of critical damping. For a full description of the structure, see [1].

The structure is subject to uncertain earthquake excitations modeled as a nonstationary stochastic process. To simulate a time history of the ground motion acceleration for given moment magnitude $M$ and epicentral distance $r$, a discrete-time white noise sequence $W_{j}=\sqrt{2 \pi / \Delta t} Z_{j}, j=1, \ldots, N_{t}$ is first generated, where

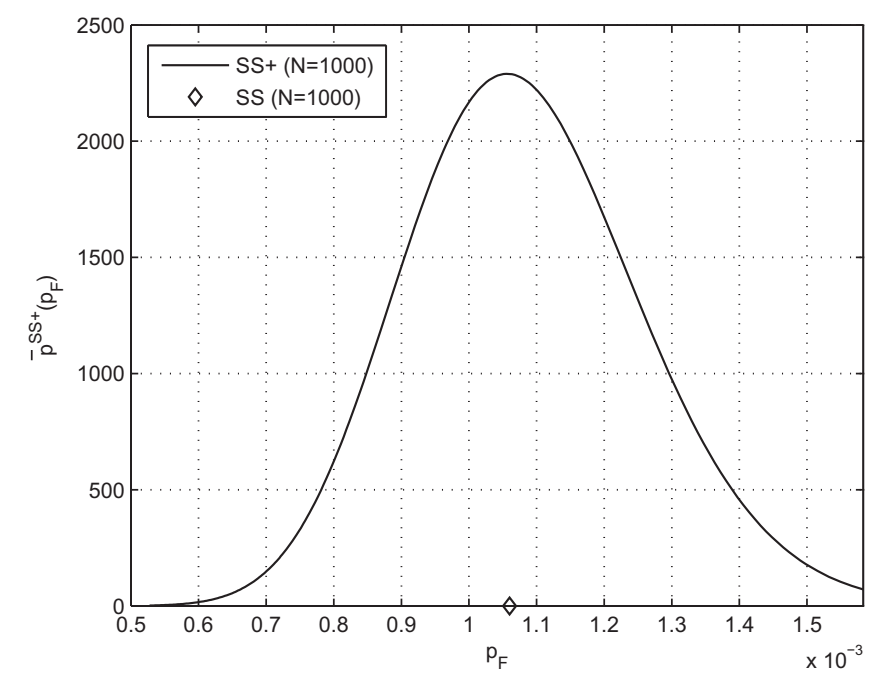

Fig. 8. The failure probability estimate $\hat{p}_{F}^{S S}$ obtained by SS and the approximation of the posterior PDF $\tilde{p}^{S S+}$ obtained by SS+. The posterior PDF has mean $\mu_{\tilde{p} S_{+}}=1.064 \times$ $10^{-3}$ and the c.o.v. $\delta_{\tilde{p}^{s s+}}=0.16$.
$\Delta t=0.03 \mathrm{~s}$ is the sampling time, $N_{t}=1001$ is the number of time instants (which corresponds to a duration of $30 \mathrm{~s}$ ), and $Z_{1}, \ldots, Z_{N_{t}}$ are i.i.d. standard Gaussian variables. The white noise sequence is then modulated (multiplied) by an envelope function $e(t ; M, r)$ at the discrete time instants. The discrete Fourier transform is then applied to the modulated white-noise sequence. The resulting spectrum is multiplied with a radiation spectrum $A(f ; M, r)[1]$, after which the discrete inverse Fourier transform is applied to transform the sequence back to time domain to yield a sample for the ground acceleration time history. The synthetic ground motion $a(t ; Z, M, r)$ generated from the model is thus a function of the Gaussian vector $Z=\left(Z_{1}, \ldots, Z_{N_{t}}\right)^{T}$ and stochastic excitation model parameters $M$ and $r$. Here, $M=7$ and $r=50 \mathrm{~km}$ are used. For more details about the ground motion sampling, refer to [1].

In this example, the uncertainty arises from seismic excitations and the uncertain parameters $\theta=Z$, the i.i.d. Gaussian sequence $Z_{1}, \ldots, Z_{N_{t}}$ that generates the synthetic ground motion. The system response of interest, $g(\theta)$, is defined to be the peak (absolute) interstory drift ratio $\delta_{\max }=\max _{i=1, \ldots, 6} \delta_{i}$, where $\delta_{i}$ is the maximum absolute interstory drift ratio of the $i$ th story within the duration of study, $30 \mathrm{~s}$. The failure domain $F \subset \mathbb{R}^{N_{t}}$ is defined as the exceedance of peak interstory drift ratio in any one of the stories within the duration of study. That is

$F=\left\{\theta \in \mathbb{R}^{N_{t}}: \delta_{\max }(\theta)>b\right\}$,

where $b$ is some prescribed critical threshold. In this example, $b=0.5 \%$ is considered, which, according to [43], corresponds to "operational" damage level. For this damage level, the structure may have a small amount of yielding.

The failure probability is estimated to be equal to $p_{F}=8.9 \times$ $10^{-3}$ (based on $4 \times 10^{4}$ Monte Carlo samples). In the application of Subset Simulation, three different implementation scenarios are considered: $N=500, N=1000$, and $N=2000$ samples are simulated at each conditional level. The failure probability estimates $\hat{p}_{F}^{S S}$ obtained by SS for these scenarios and the approximation of the corresponding posterior PDFs $\tilde{p}^{S S+}$ obtained by SS+ are given in Fig. 9. Observe that the more samples used (i.e. the more information about the system that is extracted), the more narrowly $\tilde{p}^{S S+}$ is focused around $\hat{p}_{F}^{S S}$, as expected. The coefficients of variation are $\delta_{\tilde{p} s s_{+}}=0.190, \delta_{\tilde{p} s s_{+}}=0.134$, and $\delta_{\tilde{p} s s_{+}}=0.095$ for $N=500, N=1000$, and $N=2000$, respectively. The corresponding frequentist coefficients of variation of the original SS estimator $\hat{p}_{F}^{S S}$ are $\delta_{\hat{p}_{F}^{S S}}=$

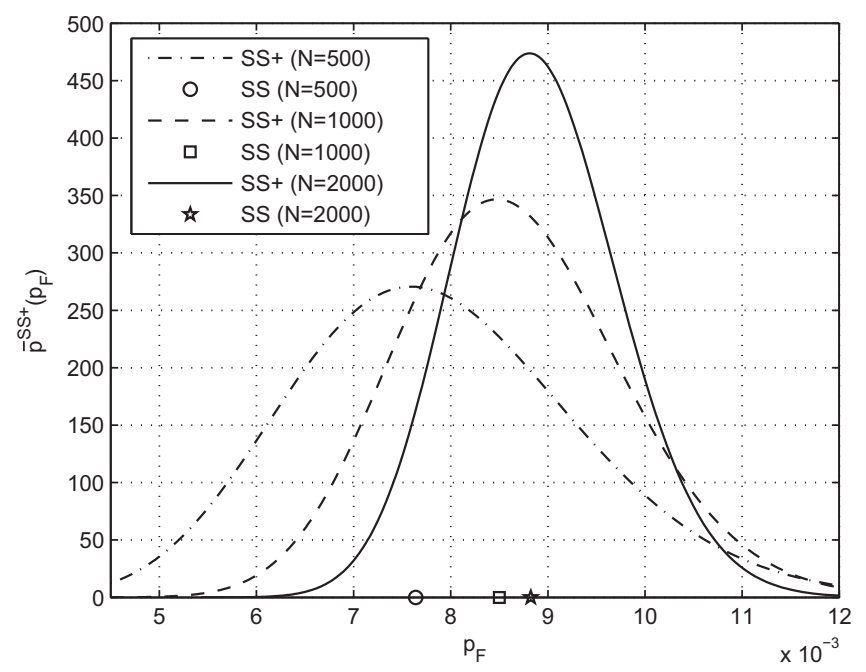

Fig. 9. The failure probability estimates $\hat{p}_{F}^{S S}$ obtained by SS and the approximation of the posterior PDF $\tilde{p}^{S S+}$ obtained by SS+ for three computational scenarios: $N=500, N=1000$, and $N=2000$ samples at each conditional level. 
$0.303, \delta_{\hat{p}_{F}^{s s}}=0.201$, and $\delta_{\hat{p}_{F}^{s s}}=0.131$ (based on 50 independent runs of the algorithm). In SS+, the coefficient of variation $\delta_{\tilde{p}^{s S+}}$ can be considered as a measure of uncertainty, based on the generated samples.

\section{Conclusions}

This paper focuses on enhancements to the Subset Simulation (SS) method, an efficient algorithm for computing failure probabilities for general high-dimensional reliability problems proposed by Au and Beck [2].

First, we explore MMA (Modified Metropolis algorithm), an MCMC technique employed within SS. This exploration leads to the following nearly optimal scaling strategy for MMA: at simulation level $j \geqslant 1$, select $\sigma_{j}$ such that the corresponding acceptance rate $\rho_{j}$ is between $30 \%$ and $50 \%$.

Next, we provide a theoretical basis for the optimal value of the conditional failure probability $p_{0}$. We demonstrate that choosing any $p_{0} \in[0.1,0.3]$ will lead to similar efficiency and it is not necessary to fine tune the value of the conditional failure probability as long as SS is implemented properly.

Finally, a Bayesian extension SS+ of the original SS method is developed. In SS+, the uncertain failure probability $p_{F}$ that one is estimating is modeled as a stochastic variable whose possible values belong to the unit interval. Instead of a single real number as an estimate as in SS, SS+ produces the posterior PDF $p^{S S+}\left(p_{F}\right)$ of the failure probability, which takes into account both prior information and the information in the samples generated by SS. This PDF quantifies the uncertainty in the value of $p_{F}$ based on the samples and prior information and it may be used in risk analyses to incorporate this uncertainty, or its approximation $\tilde{p}^{S S+}\left(p_{F}\right)$, which is more convenient for further computations. The original SS estimate corresponds to the most probable value in the Bayesian approach.

\section{Acknowledgements}

This work was supported by the National Science Foundation, under award number EAR-0941374 to the California Institute of Technology. This support is gratefully acknowledged. Any opinions, findings, and conclusions or recommendations expressed in this material are those of the authors and do not necessarily reflect those of the National Science Foundation. Partial support is acknowledged from the Hong Kong Research Grant Council through the General Research Fund 9041550 (CityU 110210).

\section{Appendix A}

In this appendix we give a detailed proof that $\pi(\cdot \mid \mathbb{E})$ is the stationary distribution for the Markov chain generated by the Modified Metropolis algorithm described in Section 2.

Theorem 3 [2]. Let $\theta^{(1)}, \theta^{(2)}, \ldots$ be the Markov chain generated by the Modified Metropolis algorithm, then $\pi(\cdot \mid \mathbb{F})$ is a stationary distribution, i.e. if $\theta^{(i)}$ is distributed according to $\pi(\cdot \mid \mathbb{E})$, then so is $\theta^{(i+1)}$.

Proof. Let $K$ denote the transition kernel of the Markov chain generated by MMA. From the structure of the algorithm it follows that $K$ has the following form:

$K\left(d \theta^{(i+1)} \mid \theta^{(i)}\right)=k\left(d \theta^{(i+1)} \mid \theta^{(i)}\right)+r\left(\theta^{(i)}\right) \delta_{\theta^{(i)}}\left(d \theta^{(i+1)}\right)$,

where $k$ describes the transitions from $\theta^{(i)}$ to $\theta^{(i+1)} \neq \theta^{(i)}, r\left(\theta^{(i)}\right)=$ $1-\int_{\mathbb{F}} k\left(d \theta^{\prime} \mid \theta^{(i)}\right)$ is the probability of remaining at $\theta^{(i)}$, and $\delta_{\theta}$ denotes point mass at $\theta$ (Dirac measure). Note that if $\theta^{(i+1)} \neq \theta^{(i)}$, then $k\left(d \theta^{(i+1)} \mid \theta^{(i)}\right)$ can be expressed as a product of the component transitional kernels:

$k\left(d \theta^{(i+1)} \mid \theta^{(i)}\right)=\prod_{j=1}^{d} k_{j}\left(d \theta_{j}^{(i+1)} \mid \theta_{j}^{(i)}\right)$,

where $k_{j}$ is the transitional kernel for the $j$ th component of $\theta^{(i)}$. By definition of the algorithm

$$
\begin{aligned}
k_{j}\left(d \theta_{j}^{(i+1)} \mid \theta_{j}^{(i)}\right)= & S_{j}\left(\theta_{j}^{(i+1)} \mid \theta_{j}^{(i)}\right) \min \left\{1, \frac{\pi_{j}\left(\theta_{j}^{(i+1)}\right)}{\pi_{j}\left(\theta_{j}^{(i)}\right)}\right\} d \theta_{j}^{(i+1)} \\
& +r_{j}\left(\theta_{j}^{(i)}\right) \delta_{\theta_{j}^{(i)}}\left(d \theta_{j}^{(i+1)}\right) .
\end{aligned}
$$

A sufficient condition for $\pi(\cdot \mid \mathbb{F})$ to be a stationary distribution for $K$ is to satisfy the so-called reversibility condition (also sometimes called the detailed balance equation):

$\pi\left(d \theta^{(i)} \mid \mathbb{F}\right) K\left(d \theta^{(i+1)} \mid \theta^{(i)}\right)=\pi\left(d \theta^{(i+1)} \mid \mathbb{F}\right) K\left(d \theta^{(i)} \mid \theta^{(i+1)}\right)$

Indeed, given (53) and $\theta^{(i)} \sim \pi(\cdot \mid \mathbb{F})$, the distribution of $\theta^{(i+1)}$ is

$$
\begin{aligned}
p\left(d \theta^{(i+1)}\right) & =\int_{\mathbb{F}} \pi\left(d \theta^{(i)} \mid \mathbb{F}\right) K\left(d \theta^{(i+1)} \mid \theta^{(i)}\right) \\
& =\int_{\mathbb{F}} \pi\left(d \theta^{(i+1)} \mid \mathbb{F}\right) K\left(d \theta^{(i)} \mid \theta^{(i+1)}\right) \\
& =\pi\left(d \theta^{(i+1)} \mid \mathbb{F}\right) \int_{\mathbb{F}} K\left(d \theta^{(i)} \mid \theta^{(i+1)}\right)=\pi\left(d \theta^{(i+1)} \mid \mathbb{F}\right),
\end{aligned}
$$

since $\int_{\mathbb{F}} K\left(d \theta^{(i)} \mid \theta^{(i+1)}\right) \equiv 1$.

So, all we need to prove is that the transition kernel $K$ of the MM algorithm satisfies the reversibility condition (53). Since all the Markov chain samples lie in $\mathbb{F}$, it is sufficient to consider the transition only between states in $\mathbb{F}$. Hence, without loss of generality, we assume that both $\theta^{(i)}$ and $\theta^{(i+1)}$ belong to $\mathbb{F}$. In addition, we assume that $\theta^{(i)} \neq \theta^{(i+1)}$, since otherwise (53) is trivial. It then follows from (50) that in this case $K\left(d \theta^{(i+1)} \mid \theta^{(i)}\right)=$ $k\left(d \theta^{(i+1)} \mid \theta^{(i)}\right)$. Taking into account (51), we can rewrite the reversibility condition (53) in terms of components:

$$
\prod_{j=1}^{d} \pi_{j}\left(\theta_{j}^{(i)}\right) k_{j}\left(d \theta_{j}^{(i+1)} \mid \theta_{j}^{(i)}\right) d \theta_{j}^{(i)}=\prod_{j=1}^{d} \pi_{j}\left(\theta_{j}^{(i+1)}\right) k_{j}\left(d \theta_{j}^{(i)} \mid \theta_{j}^{(i+1)}\right) d \theta_{j}^{(i+1)}
$$

Therefore, it is enough to show that for all $j=1, \ldots, d$

$\pi_{j}\left(\theta_{j}^{(i)}\right) k_{j}\left(d \theta_{j}^{(i+1)} \mid \theta_{j}^{(i)}\right) d \theta_{j}^{(i)}=\pi_{j}\left(\theta_{j}^{(i+1)}\right) k_{j}\left(d \theta_{j}^{(i)} \mid \theta_{j}^{(i+1)}\right) d \theta_{j}^{(i+1)}$.

If $\theta_{j}^{(i)}=\theta_{j}^{(i+1)}$, then (56) is trivial. Assume that $\theta_{j}^{(i)} \neq \theta_{j}^{(i+1)}$. Then, it follows from (52) that $k_{j}\left(d \theta_{j}^{(i+1)} \mid \theta_{j}^{(i)}\right)=S_{j}\left(\theta_{j}^{(i+1)} \mid \theta_{j}^{(i)}\right) \mathrm{min}$ $\left\{1, \frac{\pi_{j}\left(\theta_{j}^{(i+1)}\right)}{\pi_{j}\left(\theta_{j}^{(i)}\right)}\right\} d \theta_{j}^{(i+1)}$, and (56) reduces to

$$
\begin{aligned}
& \pi_{j}\left(\theta_{j}^{(i)}\right) S_{j}\left(\theta_{j}^{(i+1)} \mid \theta_{j}^{(i)}\right) \min \left\{1, \frac{\pi_{j}\left(\theta_{j}^{(i+1)}\right)}{\pi_{j}\left(\theta_{j}^{(i)}\right)}\right\} \\
& =\pi_{j}\left(\theta_{j}^{(i+1)}\right) S_{j}\left(\theta_{j}^{(i)} \mid \theta_{j}^{(i+1)}\right) \min \left\{1, \frac{\pi_{j}\left(\theta_{j}^{(i)}\right)}{\pi_{j}\left(\theta_{j}^{(i+1)}\right)}\right\} .
\end{aligned}
$$

Since $S_{j}$ is symmetric and $b \min \{1, a / b\}=a \min \{1, b / a\}$ for any positive numbers $a$ and $b,(57)$ is satisfied. This proves that $\pi(\cdot \mid \mathbb{F})$ is $a$ stationary distribution of the MMA Markov chain. 
Remark 14. A stationary distribution is unique and, therefore, is the limiting distribution for a Markov chain, if the chain is aperiodic and irreducible (see, for example, [41]). In the case of MMA, aperiodicity is guaranteed by the fact that the probability of having a repeated sample $\theta^{(i+1)}=\theta^{(i)}$ is not zero. A Markov chain with stationary distribution $p(\cdot)$ is irreducible if, for any initial state, it has positive probability of entering any set to which $p(\cdot)$ assigns positive probability. It is clear that MMA with "standard" proposal distributions (e.g. Gaussian, uniform, log-normal, etc.) generates irreducible Markov chains. In this case, $\pi(\cdot \mid \mathbb{F})$ is therefore the unique stationary distribution of the MMA Markov chain.

\section{References}

[1] Au SK. Reliability based design sensitivity by efficient simulation. Comput Struct 2005;83:1048-61.

[2] Au SK, Beck JL. Estimation of small failure probabilities in high dimensions by subset simulation. Prob Eng Mech 2001;16(4):263-77.

[3] Au SK, Beck JL. First-excursion probabilities for linear systems by very efficient importance sampling. Prob Eng Mech 2001;16(3):193-207.

[4] Au SK, Beck JL. Subset Simulation and its application to seismic risk based on dynamic analysis". J Eng Mech 2003;129(8):901-17.

[5] Au SK, Ching J, Beck JL. Application of subset simulation methods to reliability benchmark problems. Struct Safe 2007;29:183-93.

[6] Bayes T. An essay towards solving a problem in the doctrine of chances. Philos Trans Roy Soc London 1763;53:370-418 [reprinted in Biometrika 1989;45:296-315].

[7] Beck JL. Bayesian system identification based on probability logic. Struct Control Health Monit 2010;17:825-47.

[8] Bedard M, Rosenthal JS. Optimal scaling of metropolis algorithms: heading toward general target distributions. Can J Stat 2008;36:483-503.

[9] Ching J, Au SK, Beck JL. Reliability estimation of dynamical systems subject to stochastic excitation using subset simulation with splitting. Comput Methods Appl Mech Eng 2005;194(12-16):1557-79.

[10] Ching J, Beck JL, Au SK. Hybrid subset simulation method for reliability estimation of dynamical systems subject to stochastic excitation. Prob Eng Mech 2005;20(3):199-214.

[11] Cox RT. Probability, frequency, and reasonable expectation. Am J Phys 1946;14:1-13.

[12] Cox RT. The algebra of probable inference. Baltimore: The Johns Hopkins University Press; 1961

[13] Fan D-Y. The distribution of the product of independent beta variables. Commun Stat - Theor Methods 1991;20(12):4043-52.

[14] Doob JL. Stochastic processes. New York: Wiley; 1953.

[15] Galambos J, Simonelli I. Products of random variables: applications to problems of physics and to arithmetical functions. New York, Basel: Chapman \& Hall/CRC Pure and Applied Mathematics; 2004.

[16] Gelman A, Carlin JB, Stern HS, Rubin DB. Bayesian data analysis. 2nd ed. CRC Press; 2003.

[17] Gelman A, Roberts GO, Gilks WR. Efficient Metropolis jumping rules. Bayesian Stat 1996;5:599-607.

[18] Hastings WK. Monte Carlo sampling methods using Markov chains and their applications. Biometrika 1970;57(1):97-109.

[19] Jaynes ET. Information theory and statistical mechanics. Phys Rev 1957;106:620-30.

[20] Jaynes ET. In: Rosenkrantz RD, editor. Papers on probability, statistics, and statistical physics. Kluwer Academic Publishers; 1983.
[21] Jaynes ET. In: Bretthorst GL, editor. Probabiity theory: the logic of science. Cambridge University Press; 2003.

[22] Jeffreys $\mathrm{H}$. Theory of probability. 3rd revised edition 1961. Oxford: Oxford University Press; 1939.

[23] Katafygiotis LS, Cheung SH. A two-stage subset simulation-based approach for calculating the reliability of inelastic structural systems subjected to Gaussian random excitations. Comput Methods Appl Mech Eng 2005;194(1216):1581-95.

[24] Katafygiotis LS, Cheung SH. Application of spherical subset simulation method and auxiliary domain method on a benchmark reliability study. Struct Safe 2007;29(3):194-207.

[25] Katafygiotis LS, Moan T, Cheung SH. Auxiliary domain method for solving multi-objective dynamic reliability problems for nonlinear structures. Struct Eng Mech 2007;25(3):347-63.

[26] Katafygiotis LS, Zuev KM. Geometric insight into the challenges of solving high-dimensional reliability problems. Prob Eng Mech 2008;23(2-3):208-18.

[27] Koo H, Der Kiureghian A. FORM, SORM and simulation techniques for nonlinear random vibrations. Report No. UCB/SEMM-2003/01. Berkeley, CA February: Department of Civil \& Environmental Engineering, University of California; 2003

[28] Koutsourelakis P, Pradlwarter HJ, Schuëller GI. Reliability of structures in high dimensions. Part I: Algorithms and applications. Prob Eng Mech 2004;19(4):409-17.

[29] Laplace PS. Theorie analytique des probabilites. Courcier, Paris; 1812

[30] Laplace PS. Philosophical essay on probability. New York: Dover Publications; 1951.

[31] Liu JS. Monte Carlo strategies is scientific computing. New York: SpringerVerlag; 2001.

[32] Melchers R. Structural reliability analysis and prediction. Chichester: John Wiley \& Sons; 1999.

[33] Metropolis N, Rosenbluth AW, Rosenbluth MN, Teller AH, Teller E. Equation of state calculations by fast computing machines. J. Chem. Phys. 1953;21(6):1087-92.

[34] Neal RM. Probabilistic inference using Markov Chain Monte Carlo methods. Technical Report CRG-TR-93-1, Dept. of Computer Science, University of Toronto; 1993.

[35] Robert CP, Casella G. Monte Carlo statistical methods. 2nd ed. New York: Springer-Verlag; 2004.

[36] Roberts GO, Rosenthal JS. Optimal scaling of discrete approximations to Langevin diffusions. J R Stat Soc Ser B Stat Methodol 1998;60:255-68.

[37] Rohatgi VK. An introduction to probability theory and mathematical statistics. New York: Wiley; 1976.

[38] Schuëller GI. Efficient Monte Carlo simulation procedures in structural uncertainty and reliability analysis - recent advances. Struct. Eng. Mech 2009;32(1):1-20.

[39] Schuëller GI, Pradlwarter HJ, Beck JL, Au SK, Katafygiotis LS, Ghanem R. Benchmark study on reliability estimation in higher dimensions of structura systems - an overview. In: Proceedings 6th European conference on structural dynamics. Rotterdam: Millpress; 2005.

[40] Tang J, Gupta AK. On the distribution of the product of independent beta random variables. Stat Prob Lett 1984;2:165-8.

[41] Tierney L. Markov chains for exploring posterior distributions. Ann Stat 1994;22(4):1701-62.

[42] Tukey JW, Wilks SS. Approximation of the distribution of the product of beta variables by a single beta variable. Ann Math Stat 1946;17(3):318-24.

[43] Vision 2000: Performance based seismic engineering of buildings. Tech. rep. Sacramento, California: Structural Engineers Association of California, 2000.

[44] Zuev KM, Katafygiotis LS. Horseracing Simulation algorithm for evaluation of small failure probabilities. Prob Eng Mech 2011;26(2):157-64.

[45] Zuev KM, Katafygiotis LS. Modified Metropolis-Hastings algorithm with delayed rejection. Prob Eng Mech 2011;26(3):405-12. 